\title{
Piecewise partially separable functions and a derivative-free algorithm for large scale nonsmooth optimization
}

\author{
Adil M. Bagirov and Julien Ugon \\ Centre for Informatics and Applied Optimization, School of Information Technology and \\ Mathematical Sciences, University of Ballarat, Victoria 3353, Australia
}

\begin{abstract}
This paper introduces the notion of piecewise partially separable functions and studies their properties. We also consider some of many applications of these functions. Finally, we consider the problem of minimizing of piecewise partially separable functions and develop an algorithm for its solution. This algorithm exploits the structure of such functions. We present the results of preliminary numerical experiments.
\end{abstract}

Keywords: nonsmooth optimization, subdifferential, discrete gradient, large scale optimization, piecewise partially separable functions.

Mathematical Subject Classification: 65K05, 90C25.

\section{Introduction}

Some important practical problems can be reduced to nonsmooth optimization problems which contain hundreds or thousands of variables. The cluster analysis problem and the problem of calculation of piecewise linear function separating two sets are such problems (see, $[7,8,9,10,11,19,22]$ ).

Currently available general-purpose nonsmooth optimization methods are not efficient to solve such problems. To our best knowledge the paper [18] presents the first algorithm for dealing with large scale nonsmooth optimization problems. In this paper variable metric bundle algorithm with limited memory has been developed.

Large-scale optimization problems, as a rule, have a special structure. This structure is exploited to design efficient algorithms. Last two decades different algorithms have been developed for solving large scale optimization problems where both objective and constraint functions are twice continuously differentiable (see, for example, $[13,14,17]$ ). These algorithms strongly rely on the structure of large scale optimization problems, specifically the sparsity of Hessians of the objective and constraint functions.

In this paper we study large scale nonsmooth optimization problems. We introduce a class of piecewise partially separable functions and develop an algorithm for their minimization. This algorithm is based on the so-called discrete gradient method (see $[5,6]$ ). 
We present preliminary results of numerical experiments which demonstrate that the proposed algorithm is efficient for minimization of piecewise partially separable functions with several thousand variables.

The paper has the following structure. In Section 2 we introduce the new class of nonsmooth functions. Section 3 presents some properties of piecewise partially separable functions. We describe some of many applications of these functions in Section 4. We discuss an algorithm for minimizing of one subclass of piecewise partially separable functions in Section 5. Section 6 presents the results of preliminary numerical experiments and Section 7 concludes the paper.

\section{Piecewise partially separable functions: definition and ex- amples}

Let $f$ be a scalar function defined on an open set $D_{0} \subseteq \mathbb{R}^{n}$ containing a closed set $D \subseteq \mathbb{R}^{n}$. Here $\mathbb{R}^{n}$ is an $n$-dimensional Euclidean space.

Definition 1 The function $f$ is called partially separable if there exists a family of $n \times n$ diagonal matrices $U_{i}, i=1, \ldots, M$ such that the function $f$ can be represented as follows:

$$
f(x)=\sum_{i=1}^{M} f_{i}\left(U_{i} x\right) .
$$

Without loss of generality we assume that the matrices $U_{i}$ are binary, that is they contain only 0 and 1 . It is also assumed that the number $m_{i}$ of non-zero elements in the diagonal of the matrix $U_{i}$ is much smaller than $n$.

In other terms, the function $f$ is called partially separable if it can be represented as the sum of functions of a much smaller number of variables. If $M=n$ and $\operatorname{diag}\left(U_{i}\right)=e_{i}$ where $e_{i}$ is the $i-t h$ orth vector, then the function $f$ is separable.

Remark 1 Any function $f$ can be considered as partially separable if we take $M=1$ and $U_{1}=I$, where $I$ is the identity matrix. However, we consider situations where $M>1$ and $m_{i} \ll n, i=1, \ldots, M$.

Example 1 Consider the following function

$$
f(x)=\sum_{i=1}^{n} \min \left\{\left|x_{i}\right|,\left|x_{1}\right|\right\} .
$$

This function is partially separable. Indeed, in this case $M=n, m_{i}=2, U_{i}^{11}=1, U_{i}^{i i}=1$, all other elements of $U_{i}$ are zeros for all $i=1, \ldots, n$ and $f_{i}\left(U_{i} x\right)=\min \left\{\left|x_{i}\right|,\left|x_{1}\right|\right\}$.

Definition 2 The function $f$ is said to be piecewise partially separable if there exists a finite family of closed sets $D_{1}, \ldots, D_{m}$ such that $\bigcup_{i=1}^{m} D_{i}=D$ and the function $f$ is partially separable on each set $D_{i}, i=1, \ldots, m$. 
Example 2 All partially separable functions are piecewise partially separable.

Example 3 Consider the following function

$$
f(x)=\max _{j=1, \ldots, n} \sum_{i=1}^{n}\left|x_{i}-x_{j}\right| .
$$

The function $f$ is piecewise partially separable. It is clear that the functions

$$
\varphi_{j}(x)=\sum_{i=1}^{n}\left|x_{i}-x_{j}\right|, j=1, \ldots, n
$$

are partially separable with $M=n, m_{i}=2$ and $U_{i}^{i i}=U_{i}^{j j}=1$ for all $i=1, \ldots, n$. In this case the sets $D_{i}, i=1, \ldots, n$ are defined as follows:

$$
D_{i}=\left\{x \in \mathbb{R}^{n}: \varphi_{i}(x) \geq \varphi_{j}(x), \quad j=1, \ldots, n, \quad j \neq i\right\} .
$$

The piecewise partially separability of the function $f$ follows from the fact that the maximum of partially separable functions is piecewise partially separable, which will be proved later on in Proposition 7.

\subsection{Chained and piecewise chained functions}

One of the interesting and important classes of partially separable functions is the one of the so-called chained functions.

Definition 3 The function $f$ is said to be $k$-chained, $k \leq n$, if it can be represented as follows:

$$
f(x)=\sum_{i=1}^{n-k+1} f_{i}\left(x_{i}, \ldots, x_{i+k-1}\right) .
$$

For example, if $k=2$, the function $f$ is:

$$
f(x)=\sum_{i=1}^{n-1} f_{i}\left(x_{i}, x_{i+1}\right) .
$$

Proposition 1 Any k-chained function is partially separable.

Proof: Indeed for $k$-chained functions $M=n-k+1, m_{i}=k$ and the matrices $U_{i}, i=$ $1, \ldots, M$ are defined as follows:

$$
U_{i}^{j j}=1, j=i, \ldots, i+k-1
$$

and all other elements of $U_{i}$ are zeros.

Proposition 2 Any separable function is 1-chained. 
Definition 4 The function $f$ is said to be piecewise $k$-chained if there exists a finite family of closed sets $D_{1}, \ldots, D_{m}$ such that $\bigcup_{i=1}^{m} D_{i}=D$ and the function $f$ is $k$-chained on each set $D_{i}, i=1, \ldots, m$.

Proposition 3 Any piecewise $k$-chained function is piecewise partially separable.

The proof directly follows from Proposition 1.

The following is an example of piecewise 2-chained function.

Example 4 (Chained Crescent I function ([25])

$$
f(x)=\max \left\{f_{1}(x), f_{2}(x)\right\}
$$

where

$$
\begin{gathered}
f_{1}(x)=\sum_{i=1}^{n-1}\left(x_{i}^{2}+\left(x_{i+1}-1\right)^{2}+x_{i+1}-1\right), \\
f_{2}(x)=\sum_{i=1}^{n-1}\left(-x_{i}^{2}-\left(x_{i+1}-1\right)^{2}+x_{i+1}+1\right) .
\end{gathered}
$$

Both $f_{1}$ and $f_{2}$ are 2-chained functions. We define two sets as follows:

$$
\begin{aligned}
& D_{1}=\left\{x \in \mathbb{R}^{n}: f_{1}(x) \geq f_{2}(x)\right\}, \\
& D_{2}=\left\{x \in \mathbb{R}^{n}: f_{2}(x) \geq f_{1}(x)\right\} .
\end{aligned}
$$

It is clear that the sets $D_{1}, D_{2}$ are closed, $f(x)=f_{1}(x)$ for $x \in D_{1}$ and $f(x)=f_{2}(x)$ for $x \in D_{2}$. Furthermore $D_{1} \cup D_{2}=D$. Thus the function $f$ is piecewise 2-chained.

\subsection{Piecewise separable functions}

Definition 5 The function $f$ is said to be piecewise separable if there exists a finite family of closed sets $D_{1}, \ldots, D_{m}$ such that $\bigcup_{i=1}^{m} D_{i}=D$ and the function $f$ is separable on each set $D_{i}, i=1, \ldots, m$.

Proposition 4 Any piecewise separable function is piecewise 1-chained.

Proof: Since any separable function is 1-chained (Proposition 2) the proof is straightforward.

Corollary 1 Any piecewise separable function is piecewise partially separable.

Proposition 5 All separable functions are piecewise separable. In this case $m=1$.

Example 5 All piecewise linear functions are piecewise separable. A function $f: D \rightarrow \mathbb{R}^{1}$ is said to be piecewise linear if there exists a finite family of closed sets $Q_{1}, \ldots, Q_{p}$ such that $\bigcup_{i=1}^{p} Q_{i}=D$ and the function $f$ is linear on each set $Q_{i}, i=1, \ldots, p$. Since any linear function is separable the function $f$ is piecewise separable and in this case $m=p$. $\triangle$ 
Example 6 One of the simplest piecewise separable functions is the following maximum function:

$$
f(x)=\max _{i=1, \ldots, n} x_{i}^{2} .
$$

Here $m=n$ and

$$
D_{i}=\left\{x \in \mathbb{R}^{n}: x_{i}^{2} \geq x_{j}^{2}, j=1, \ldots, n, j \neq i\right\} .
$$

$f(x)=x_{i}^{2}$ for any $x \in D_{i}$. It is clear that $\bigcup_{i=1}^{m} D_{i}=\mathbb{R}^{n}$. It should be noted that the function $f$ is neither separable nor piecewise linear.

\section{Properties of piecewise partially separable functions}

In this section we study some properties of piecewise partially separable functions.

Proposition 6 Let $f_{1}$ and $f_{2}$ be partially separable functions on the closed D. Then the function $f(x)=f_{1}(x)+f_{2}(x)$ is also partially separable on $D$.

Proof: Since the functions $f_{1}$ and $f_{2}$ are partially separable there exist families of matrices $U_{i}^{1}, i=1, \ldots, M_{1}$ and $U_{j}^{2}, j=1, \ldots, M_{2}$ such that

$$
\begin{aligned}
& f_{1}(x)=\sum_{i=1}^{M_{1}} f_{1 i}\left(U_{i}^{1} x\right), \\
& f_{2}(x)=\sum_{j=1}^{M_{2}} f_{2 j}\left(U_{j}^{2} x\right) .
\end{aligned}
$$

Consider the following sets:

$$
\begin{gathered}
I=\left\{i \in\left\{1, \ldots, M_{1}\right\}: U_{i}^{1} \neq U_{j}^{2}, \forall j \in\left\{1, \ldots, M_{2}\right\}\right\}, \\
J=\left\{j \in\left\{1, \ldots, M_{2}\right\}: U_{j}^{2} \neq U_{i}^{1}, \forall i \in\left\{1, \ldots, M_{1}\right\}\right\}, \\
H=\left\{(i, j), i \in\left\{1, \ldots, M_{1}\right\}, j \in\left\{1, \ldots, M_{2}\right\}: U_{i}^{1}=U_{j}^{2}\right\} .
\end{gathered}
$$

It is clear that for any $i \in I$ there is no $j \in\left\{1, \ldots, M_{2}\right\}$ such that $(i, j) \in H$ and similarly for any $j \in J$ there is no $i \in\left\{1, \ldots, M_{1}\right\}$ such that $(i, j) \in H$. Then the function $f$ can be represented as follows

$$
f(x)=\sum_{(i, j) \in H}\left(f_{1 i}\left(U_{i}^{1} x\right)+f_{2 j}\left(U_{j}^{2} x\right)\right)+\sum_{i \in I} f_{1 i}\left(U_{i}^{1} x\right)+\sum_{j \in J} f_{2 j}\left(U_{j}^{2} x\right) .
$$

This function is partially separable, that is

$$
f(x)=\sum_{k=1}^{M} \bar{f}_{k}\left(V_{k} x\right),
$$


where $M=M_{1}+M_{2}-\operatorname{card}(H)$, the matrices $V_{k}, k=1, \ldots, M$ can be defined as follows:

$$
V_{k}=\left\{\begin{array}{lll}
U_{i}^{1}=U_{j}^{2} & k=1, \ldots, \operatorname{card}(H) & (i, j) \in H \\
U_{i}^{1} & k=\operatorname{card}(H)+1, \ldots, M_{1} & i \in I \\
U_{j}^{2} & k=M_{1}+1, \ldots, M_{1}+M_{2}-\operatorname{card}(H) & j \in J
\end{array}\right.
$$

and

$$
\bar{f}_{k}\left(V_{k} x\right)=\left\{\begin{array}{lll}
\left(f_{1 i}\left(U_{i}^{1} x\right)+f_{2 j}\left(U_{j}^{2} x\right)\right) & k=1, \ldots, \operatorname{card}(H) & (i, j) \in H \\
f_{1 i}\left(U_{i}^{1} x\right) & k=\operatorname{card}(H)+1, \ldots, M_{1} & i \in I \\
f_{2 j}\left(U_{j}^{2} x\right) & k=M_{1}+1, \ldots, M_{1}+M_{2}-\operatorname{card}(H) & j \in J .
\end{array}\right.
$$

Here $\operatorname{card}(H)$ stands for the cardinality of the set $H$.

We say that two partially separable functions $f_{1}$ and $f_{2}$ have the same structure if $I=J=\emptyset$. These functions are more interesting from a practical point of view. In this case the function $f$ has the same structure as $f_{1}$ and $f_{2}$ and

$$
f(x)=\sum_{(i, j) \in H}\left(f_{1 i}\left(U_{i}^{1} x\right)+f_{2 j}\left(U_{j}^{2} x\right)\right) .
$$

For example, if $f_{1}$ and $f_{2}$ are $k$-chained then the function $f$ is also $k$-chained.

Proposition 7 If $f$ and $g$ are piecewise partially separable (piecewise $k$-chained, piecewise separable) continuous functions on the closed set $D$, then

1) $h(x)=\alpha f(x), \alpha \in \mathbb{R}^{1}$ is piecewise partially separable (piecewise $k$-chained, piecewise separable);

2) $h(x)=f(x)+g(x)$ is piecewise partially separable (piecewise $k$-chained, piecewise separable);

3) $h(x)=\max (f(x), g(x)), h(x)=\min (f(x), g(x))$ and $h(x)=|f(x)|$ are piecewise partially separable (piecewise $k$-chained, piecewise separable).

Proof: 1) The proof is straightforward.

2) Since the functions $f$ and $g$ are piecewise partially separable there exist families of closed sets

$$
D_{i}^{f}, i=1, \ldots, m_{1}, \bigcup_{i=1}^{m_{1}} D_{i}^{f}=D
$$

and

$$
D_{j}^{g}, j=1, \ldots, m_{2}, \bigcup_{j=1}^{m_{2}} D_{j}^{g}=D
$$


such that the function $f$ is partially separable on the sets $D_{i}^{f}$ and the function $g$ is partially separable on the sets $D_{j}^{g}$. We define a family of sets $Q_{i j}, i=1, \ldots, m_{1}, \quad j=1, \ldots, m_{2}$ where

$$
Q_{i j}=D_{i}^{f} \bigcap D_{j}^{g}
$$

It is clear that

$$
\bigcup_{i, j} Q_{i j}=D
$$

and the sets $Q_{i j}$ are closed. Since the sum of partially separable functions is partially separable we get that $f+g$ is partially separable on each set $Q_{i j}$.

The proof for piecewise $k$-chained and piecewise separable functions is similar.

3) Consider the following two sets:

$$
P_{1}=\{x \in D: f(x) \geq g(x)\}, \quad P_{2}=\{x \in D: g(x) \geq f(x)\} .
$$

It is clear that $P_{1} \cup P_{2}=D$. Since the functions $f$ and $g$ are continuous the sets $P_{1}$ and $P_{2}$ are closed. We define the following families of sets:

$$
Q_{i}^{1}=P_{1} \bigcap D_{i}^{f}, i=1, \ldots, m_{1}, \quad Q_{j}^{2}=P_{2} \bigcap D_{j}^{g}, j=1, \ldots, m_{2} .
$$

These sets are closed. It can be easily shown that

$$
\left(\bigcup_{i}^{m_{1}} Q_{i}^{1}\right) \bigcup\left(\bigcup_{j}^{m_{2}} Q_{j}^{2}\right)=D .
$$

$h(x)=f(x), x \in Q_{i}^{1}, i=1, \ldots, m_{1}$ and $f$ is partially separable on each set $Q_{i}^{1}$. Similarly $h(x)=g(x), x \in Q_{j}^{2}, j=1, \ldots, m_{2}$ and $g$ is partially separable on each set $Q_{j}^{2}$. Then we get that the function $h$ is piecewise partially separable.

Since $h(x)=\min (f(x), g(x))=-\max (-f(x),-g(x))$ then we get that $h$ is piecewise partially separable. $h(x)=|f(x)|=\max (f(x),-f(x))$ and both $f$ and $-f$ are piecewise partially separable it follows that the function $h$ is also piecewise partially separable.

Again the proof for piecewise $k$-chained and piecewise separable functions is similar. $\triangle$

The problem of computation of Hessians of twice continuously differentiable partially separable functions was discussed by many authors (see, for example, $[1,13]$ ).

In order to describe some differential properties of piecewise partially separable functions we recall some definitions from nonsmooth analysis.

We consider a locally Lipschitz continuous function $f$ defined on $\mathbb{R}^{n}$. This function is differentiable almost everywhere and one can define for it a Clarke subdifferential (see [12]), by

$$
\partial f(x)=\operatorname{co}\left\{v \in \mathbb{R}^{n}: \exists\left(x^{k} \in D(f), x^{k} \rightarrow x, k \rightarrow+\infty\right): v=\lim _{k \rightarrow+\infty} \nabla f\left(x^{k}\right)\right\},
$$

here $D(f)$ denotes the set where $f$ is differentiable and co is a convex hull of a set. 
The function $f$ is differentiable at the point $x \in \mathbb{R}^{n}$ with respect to the direction $g \in \mathbb{R}^{n}$ if the limit

$$
f^{\prime}(x, g)=\lim _{\alpha \rightarrow+0} \frac{f(x+\alpha g)-f(x)}{\alpha}
$$

exists. The number $f^{\prime}(x, g)$ is said to be the derivative of the function $f$ with respect to the direction $g \in \mathbb{R}^{n}$ at the point $x$.

The Clarke upper derivative $f^{0}(x, g)$ of the function $f$ at the point $x$ with respect to the direction $g \in \mathbb{R}^{n}$ is defined as follows:

$$
f^{0}(x, g)=\limsup _{\alpha \rightarrow+0, y \rightarrow x} \frac{f(y+\alpha g)-f(y)}{\alpha} .
$$

The following is true (see [12])

$$
f^{0}(x, g)=\max \{\langle v, g\rangle: v \in \partial f(x)\} .
$$

Here $\langle\cdot, \cdot\rangle$ stands for an inner product in $\mathbb{R}^{n}$. It should be noted that the Clarke upper derivative always exists for locally Lipschitz continuous functions. The function $f$ is said to be regular at the point $x \in \mathbb{R}^{n}$ if

$$
f^{\prime}(x, g)=f^{0}(x, g)
$$

for all $g \in \mathbb{R}^{n}$. For Clarke regular functions there exists a calculus (see $[12,15]$ ). However in general for non-regular functions such a calculus does not exist.

Now let us assume that the function $f$ is partially separable and the functions $f_{i}, i=$ $1, \ldots, M$ are directionally differentiable. Then the function $f$ is also directionally differentiable and

$$
f^{\prime}(x, g)=\sum_{i=1}^{M} f_{i}^{\prime}\left(U_{i} x, U_{i} g\right) .
$$

It follows from this formula that if $f$ separable then

$$
f^{\prime}(x, g)=\sum_{i=1}^{n} f_{i}^{\prime}\left(x_{i}, g_{i}\right)
$$

where

$$
f_{i}^{\prime}\left(x_{i}, g_{i}\right)= \begin{cases}f_{i+}^{\prime}\left(x_{i}\right) & \text { if } g_{i}>0 \\ 0 & \text { if } g_{i}=0 \\ -f_{i-}^{\prime}\left(x_{i}\right) & \text { if } g_{i}<0\end{cases}
$$

and $f_{i+}^{\prime}\left(x_{i}\right), f_{i-}^{\prime}\left(x_{i}\right)$ are the right and left side derivatives of the function $f_{i}$ at the point $x_{i}$.

Below we study the Lipschitz continuity and directional differentiability of piecewise partially separable functions.

Let $f$ be a piecewise partially separable function on the closed convex set $D \subset \mathbb{R}^{n}$, that is there exists a family of closed sets $D_{j}, j=1, \ldots, m$ such that $\bigcup_{j=1}^{m} D_{j}=D$, $f(x)=f_{j}(x), x \in D_{j}$ and the functions $f_{j}$ are partially separable on $D_{j}$. 
Proposition 8 Let $f$ be continuous and each function $f_{j}$ be locally Lipschitz continuous on $D_{j}, j=1, \ldots, m$. Then the function $f$ is locally Lipschitz continuous on $D$.

Proof: We take any bounded subset $\bar{D} \subset D$. Then there exists a subset of indices $\left\{j_{1}, \ldots, j_{p}\right\} \subset\{1, \ldots, m\}$ such that

$$
\operatorname{co} \bar{D} \bigcap D_{j_{k}} \neq \emptyset, k=1, \ldots, p
$$

Let $L_{j_{k}}>0$ be a Lipschitz constant of the function $f_{j_{k}}$ on the set co $\bar{D} \cap D_{j_{k}}, k=1, \ldots, p$. Let

$$
L_{0}=\max _{k=1, \ldots, p} L_{j_{k}}
$$

Now we take any two points $x, y \in \bar{D}$. Then there exist indices $j_{k_{1}}, j_{k_{2}} \in\left\{j_{1}, \ldots, j_{p}\right\}$ such that $x \in D_{j_{k_{1}}}$ and $y \in D_{j_{k_{2}}}$. If $k_{1}=k_{2}=k$ then it is clear that

$$
|f(x)-f(y)|=\left|f_{k}(x)-f_{k}(y)\right| \leq L_{k}\|x-y\| \leq L_{0}\|x-y\| .
$$

Otherwise we consider the segment $[x, y]=\alpha x+(1-\alpha) y, \alpha \in[0,1]$ joining these two points and define the following set:

$$
Z_{[x, y]}=\left\{z \in[x, y]: \exists l_{1}, l_{2} \in\{1, \ldots, p\}: z \in D_{j_{l_{1}}} \bigcap D_{j_{l_{2}}}\right\}
$$

It is clear that in this case the set $Z_{[x, y]}$ is not empty. Then there exists a sequence of points $\left\{z_{1}, \ldots, z_{N}\right\} \subset Z_{[x, y]}, N \leq p$ such that

- $\left\{x, z_{1}\right\} \subset D_{j_{1}}, l_{0}=k_{1}$

- $\left\{z_{N}, y\right\} \subset D_{j_{k_{2}}}, l_{N}=k_{2}$;

- $\forall i \in\{1, \ldots, N-1\}, \exists l_{i} \in\{1, \ldots, p\}:\left\{z_{i}, z_{i+1}\right\} \subset D_{j_{l_{i}}}$.

Then taking into account the continuity of the function $f$ we have:

$$
\begin{aligned}
|f(y)-f(x)| & =\left|f(y)+\sum_{i=1}^{N}\left(f\left(z_{i}\right)-f\left(z_{i}\right)\right)-f(x)\right| \\
& =\left|f_{j_{k_{2}}}(y)+\sum_{i=1}^{N}\left(f_{j_{l_{i-1}}}\left(z_{i}\right)-f_{j_{l_{i}}}\left(z_{i}\right)\right)-f_{j_{k_{1}}}(x)\right| \\
& \leq\left|f_{j_{k_{2}}}(y)-f_{j_{k_{2}}}\left(z_{N}\right)\right|+\sum_{i=1}^{N-1}\left|f_{j_{l_{i}}}\left(z_{i+1}\right)-f_{j_{l_{i}}}\left(z_{i}\right)\right|+\left|f_{j_{k_{1}}}\left(z_{1}\right)-f_{j_{k_{1}}}(x)\right| \\
& \leq L_{j_{1}}\left\|y-z_{N}\right\|+\sum_{i=1}^{N-1} L_{j_{i}}\left\|z_{i}-z_{i+1}\right\|+L_{j_{k_{1}}}\left\|z_{1}-x\right\| \\
& \leq L_{0}\left(\left\|y-z_{N}\right\|+\sum_{i=1}^{N-1}\left\|z_{i}-z_{i+1}\right\|+\left\|z_{1}-x\right\|\right) .
\end{aligned}
$$


Then, as all $z_{i}$ are aligned on the segment $[x, y]$, we get

$$
|f(y)-f(x)| \leq L_{0}\|y-x\| .
$$

Since points $x$ and $y$ are arbitrary it follows that the function $f$ is locally Lipschitz continuous.

Corollary 2 Assume that all conditions of Proposition 8 are satisfied. Then the function $f$ is Clarke subdifferentiable.

Proposition 9 Assume that for any two points $x, y \in D$ the set $Z_{[x, y]}$ is finite and all functions $f_{j}, j=1, \ldots, m$ are directionally differentiable. Then the function $f$ is also directionally differentiable.

Proof: We take any point $x \in D$ and any direction $g \neq 0$ such that $x+\alpha g \in D, \alpha \in[0, \bar{\alpha}]$ for some $\bar{\alpha}>0$. By the definition

$$
f^{\prime}(x, g)=\lim _{\alpha \rightarrow+0} \frac{f(x+\alpha g)-f(x)}{\alpha} .
$$

Assume that $x \in \bigcap_{k \in K} D_{k}$, where $K \subset\{1, \ldots, m\}$. Let $y=x+\bar{\alpha} g \in D$. Since the set $Z_{[x, y]}$ is finite there exists a finite sequence of numbers $\alpha_{1}, \ldots, \alpha_{l}$ such that $\alpha_{i} \in(0, \bar{\alpha})$ and $x+\alpha_{j} g \in D_{k_{j}} \cap D_{k_{j+1}}, j=1, \ldots, l$ and

- $\left[x, x+\alpha_{1} g\right] \subset D_{k_{1}}, k_{1} \in K$;

- $\left[x+\alpha_{l} g, y\right] \subset D_{k_{l+1}}$;

- $\forall i \in\{1, \ldots, l-1\}:\left[x+\alpha_{i} g, x+\alpha_{i+1} g\right] \subset D_{k_{i+1}}$.

This implies that the segment $\left[x, x+\alpha_{1} g\right] \subset D_{k_{1}}$. Thus

$$
f^{\prime}(x, g)=f_{k_{1}}^{\prime}(x, g) \text {. }
$$

It follows that if the function $f$ is piecewise partially separable then its directional derivative can be calculated using (1) and if this function is piecewise separable then its directional derivative is calculated using (2).

In general piecewise partially separable functions are not regular. The following example demonstrates it.

Example 7 Consider the function

$$
f\left(x_{1}, x_{2}\right)=\max \left\{\left|x_{1}\right|-\left|x_{2}\right|,-\left|x_{1}\right|+\mid x_{2}\right\}, \quad\left(x_{1}, x_{2}\right) \in \mathbb{R}^{2} .
$$

This function is piecewise separable. However it is not regular. Indeed, for the direction $g=(1,1)$ at the point $x=(0,1)$ we have

$$
f^{\prime}(x, g)=0 \text { and } f^{0}(x, g)=2,
$$

that is $f^{\prime}(x, g)<f^{0}(x, g)$.

This example shows that in general for the subdifferential of piecewise partially separable functions a full calculus does not exist. Therefore in many cases the computation of their subgradients is quite difficult task. 


\section{Motivation: examples from applications}

In this section we present two very important applications of piecewise partially separable functions.

\subsection{Clustering function}

Cluster analysis has found many applications, including information retrieval, medicine etc. Clustering is also known as the unsupervised classification of patterns. The clustering problem has been studied by many authors and different algorithms have been developed for its solution (see [19, 22]).

In cluster analysis we assume that we have been given a finite set of points $A$ in the $n$-dimensional space $\mathbb{R}^{n}$, that is

$$
A=\left\{a^{1}, \ldots, a^{M}\right\}, \text { where } a^{i} \in \mathbb{R}^{n}, i=1, \ldots, M .
$$

The cluster analysis deals with the problems of organization of a collection of patterns $a^{i}$ into clusters based on similarity. As a measure of similarity different distances can be used. Here for the sake of simplicity we consider Euclidean distance. We consider partition clustering, that is the distribution of the points of the set $A$ into a given number $q$ of disjoint subsets $A^{i}, i=1, \ldots, q$ with respect to predefined criteria such that:

1) $A^{i} \neq \emptyset, i=1, \ldots, q$;

2) $A^{i} \cap A^{j}=\emptyset, i, j=1, \ldots, q, i \neq j$;

3) $A=\bigcup_{i=1}^{q} A^{i}$.

The sets $A^{i}, i=1, \ldots, q$ are called clusters. We can assume that each cluster $A^{i}, i=$ $1, \ldots, q$ can be identified by its center (or centroid). Then the clustering problem can be reduced to the following nonsmooth optimization problem (see [8]):

$$
\text { minimize } f\left(x^{1}, \ldots, x^{q}\right) \text { subject to }\left(x^{1}, \ldots, x^{q}\right) \in \mathbb{R}^{n \times q},
$$

where

$$
f\left(x^{1}, \ldots, x^{q}\right)=\frac{1}{M} \sum_{i=1}^{M} \min _{s=1, \ldots, q}\left\|x^{s}-a^{i}\right\|^{2} .
$$

$x^{i}$ is the center of the cluster $A^{i}, i=1, \ldots, q$. If $q>1$, the function (4) is nonconvex and nonsmooth. The problem (3) is also known as the sum-of-squares clustering problem.

It is clear that the function

$$
\psi(y)=\|y-a\|^{2}, y \in \mathbb{R}^{n}
$$

is separable and therefore the function

$$
\varphi_{i}(x)=\min _{s=1, \ldots, q}\left\|x^{s}-a^{i}\right\|^{2}
$$

is piecewise separable. Then it follows from Proposition 7 that the function (4) is piecewise separable. 


\subsection{Max-min separability}

The problems of supervised data classification arise in many areas including management science, medicine, chemistry. The aim of supervised data classification is to establish rules for the classification of some observations assuming that the classes of data are known. To find these rules, known training subsets of the given classes are used. This problem can be reduced to a number of set separation problems. For each class, the training points belonging to this class have to be separated from the other training points using a certain, not necessarily linear, function. In the paper [7] an algorithm for calculation of piecewise linear functions separating two sets is developed. This problem is formulated as a nonsmooth optimization problem with max-min-type objective function. We will briefly describe this problem.

Let $A$ and $B$ be given disjoint sets containing $m$ and $p n$-dimensional vectors, respectively:

$$
\begin{gathered}
A=\left\{a^{1}, \ldots, a^{m}\right\}, a^{i} \in \mathbb{R}^{n}, i=1, \ldots, m, \\
B=\left\{b^{1}, \ldots, b^{p}\right\}, b^{j} \in \mathbb{R}^{n}, j=1, \ldots, \quad A \bigcap B=\emptyset .
\end{gathered}
$$

Let $H=\left\{h_{1}, \ldots, h_{l}\right\}$, where $h_{j}=\left\{x^{j}, y_{j}\right\}, j=1, \ldots, l$ with $x^{j} \in \mathbb{R}^{n}, y_{j} \in \mathbb{R}^{1}$, be a finite set of hyperplanes. Let $J=\{1 \ldots, l\}$. Consider any partition of this set $J^{r}=\left\{J_{1}, \ldots, J_{r}\right\}$ such that

$$
J_{k} \neq \emptyset, k=1, \ldots, r, \quad J_{k} \bigcap J_{j}=\emptyset, \quad \bigcup_{k=1}^{r} J_{k}=J .
$$

Let $I=\{1, \ldots, r\}$. A particular partition $J^{r}=\left\{J_{1}, \ldots, J_{r}\right\}$ of the set $J$ defines the following max-min-type function:

$$
\varphi(z)=\max _{i \in I} \min _{j \in J_{i}}\left\{\left\langle x^{j}, z\right\rangle-y_{j}\right\}, z \in \mathbb{R}^{n} .
$$

Definition 6 (see [7]). The sets $A$ and $B$ are max-min separable if there exist a finite number of hyperplanes $\left\{x^{j}, y_{j}\right\}$ with $x^{j} \in \mathbb{R}^{n}, y_{j} \in \mathbb{R}^{1}, j \in J=\{1, \ldots, l\}$ and a partition $J^{r}=\left\{J_{1}, \ldots, J_{r}\right\}$ of the set $J$ such that

1) for all $i \in I$ and $a \in A$

$$
\min _{j \in J_{i}}\left\{\left\langle x^{j}, a\right\rangle-y_{j}\right\}<0
$$

2) for any $b \in B$ there exists at least one $i \in I$ such that

$$
\min _{j \in J_{i}}\left\{\left\langle x^{j}, b\right\rangle-y_{j}\right\}>0 \text {. }
$$

Remark 2 It follows from Definition 6 that if the sets $A$ and $B$ are max-min separable then $\varphi(a)<0$ for any $a \in A$ and $\varphi(b)>0$ for any $b \in B$, where the function $\varphi$ is defined by (5). Thus the sets $A$ and $B$ can be separated by a function represented as a max-min of linear functions. Therefore this kind of separability is called a max-min separability. 
The problem of the max-min separability is reduced to the following mathematical programming problem (see [7]):

$$
\text { minimize } f(x, y) \text { subject to }(x, y) \in \mathbb{R}^{l n} \times \mathbb{R}^{l}
$$

where the objective function $f$ has the following form:

$$
f(x, y)=f_{1}(x, y)+f_{2}(x, y)
$$

and

$$
\begin{aligned}
& f_{1}(x, y)=\frac{1}{m} \sum_{k=1}^{m} \max \left[0, \max _{i \in I} \min _{j \in J_{i}}\left\{\left\langle x^{j}, a^{k}\right\rangle-y_{j}+1\right\}\right], \\
& f_{2}(x, y)=\frac{1}{p} \sum_{t=1}^{p} \max \left[0, \min _{i \in I} \max _{j \in J_{i}}\left\{-\left\langle x^{j}, b^{t}\right\rangle+y_{j}+1\right\}\right] .
\end{aligned}
$$

One can see that both functions $f_{1}$ and $f_{2}$ are piecewise linear, therefore the resulting function $f$ is piecewise linear and consequently piecewise separable.

\section{Minimization of piecewise partially separable functions}

In this section we will develop an algorithm for minimizing one class of piecewise partially separable functions.

We will consider the following unconstrained minimization problem

$$
\text { minimize } f(x) \text { subject to } x \in \mathbb{R}^{n}
$$

where the objective function $f$ is as follows

$$
f(x)=\sum_{i=1}^{M} \max _{j \in J_{i}} \min _{k \in K_{j}} f_{i j k}(x)
$$

and functions $f_{i j k}, i=1, \ldots, M, j \in J_{i}, k \in K_{j}$ are partially separable, that is there exists a family of $n \times n$ matrices $U_{i j k t}, t=1, \ldots, M_{i j k}$ such that

$$
f_{i j k}(x)=\sum_{t=1}^{M_{i j k}} f_{i j k}^{t}\left(U_{i j k t} x\right) .
$$

The function $f$ is piecewise partially separable. If all functions $f_{i j k}$ are $l$-chained (separable) then the function $f$ is piecewise $l$-chained (piecewise separable).

Particular cases of this function are the following:

1. The case when the sets $J_{i}, i=1, \ldots, M$ are singletons

$$
f(x)=\sum_{i=1}^{M} \min _{k \in K_{i}} f_{i k}(x) .
$$

The clustering function serves as an example for this type of functions when $K_{i}=$ $\{1, \ldots, K\}, \forall i \in\{1, \ldots, M\}$ and the functions $f_{i k}$ are separable. 
2. The case when $M=1$

$$
f(x)=\max _{j \in J} \min _{k \in K_{j}} f_{j k}(x) .
$$

As we can see from Example 7 even for very simple cases this type of functions may not be regular and therefore sometimes the computation of their subgradients is quite difficult. Therefore, methods based on function evaluations only seem better alternatives to solve problem (9). However the existing direct search methods, including Powell method (see [28]) and Nelder-Mead simplex method [27], become inefficient when the number of variables increases.

We will develop a new modified version of the discrete gradient method for solving problem (9). This is a derivative-free method. The description of this method can be found in $[4,6]$ (see, also, [5]). The discrete gradient method can be considered as a version of the bundle method $([20,21,25])$, where subgradients of the objective function are replaced by its discrete gradients. This method consists of three main steps: the calculation of discrete gradients, the calculation of descent directions and line search. Numerical experiments have shown that for large scale problems the first step takes most of the CPU time used by the method. We will introduce a new scheme for the calculation of discrete gradients of piecewise partially separable functions represented as a sum of max-min functions. To calculate the discrete gradients we use only values of the objective function. Since the calculation of the objective function in the problem (9) can be expensive, such a scheme will allow one to significantly reduce the number of objective function evaluations.

In order to describe a new scheme for the calculation of the discrete gradient we recall here its definition.

\subsection{Discrete gradient}

Let $f$ be a locally Lipschitz continuous function defined on $\mathbb{R}^{n}$. Let

$$
\begin{gathered}
S_{1}=\left\{g \in \mathbb{R}^{n}:\|g\|=1\right\}, \quad G=\left\{e \in \mathbb{R}^{n}: e=\left(e_{1}, \ldots, e_{n}\right),\left|e_{j}\right|=1, j=1, \ldots, n\right\}, \\
P=\left\{z(\lambda): z(\lambda) \in \mathbb{R}^{1}, z(\lambda)>0, \lambda>0, \lambda^{-1} z(\lambda) \rightarrow 0, \lambda \rightarrow 0\right\}, \\
I(g, \alpha)=\left\{i \in\{1, \ldots, n\}:\left|g_{i}\right| \geq \alpha\right\},
\end{gathered}
$$

where $\alpha \in\left(0, n^{-1 / 2}\right]$ is a fixed number.

Here $S_{1}$ is the unit sphere, $G$ is the set of vertices of the unit hypercube in $\mathbb{R}^{n}$ and $P$ is the set of univariate positive infinitesimal functions.

We define operators $H_{i}^{j}: \mathbb{R}^{n} \rightarrow \mathbb{R}^{n}$ for $i=1, \ldots, n, j=0, \ldots, n$ by the formula

$$
H_{i}^{j} g= \begin{cases}\left(g_{1}, \ldots, g_{j}, 0, \ldots, 0\right) & \text { if } j<i \\ \left(g_{1}, \ldots, g_{i-1}, 0, g_{i+1}, \ldots, g_{j}, 0, \ldots, 0\right) & \text { if } j \geq i .\end{cases}
$$

We can see that

$$
H_{i}^{j} g-H_{i}^{j-1} g=\left\{\begin{array}{cl}
\left(0, \ldots, 0, g_{j}, 0, \ldots, 0\right) & \text { if } j=1, \ldots, n, j \neq i \\
0 & \text { if } j=i .
\end{array}\right.
$$


Let $e(\beta)=\left(\beta e_{1}, \beta^{2} e_{2}, \ldots, \beta^{n} e_{n}\right)$, where $\beta \in(0,1]$. For $x \in \mathbb{R}^{n}$ we consider vectors

$$
x_{i}^{j} \equiv x_{i}^{j}(g, e, z, \lambda, \beta)=x+\lambda g-z(\lambda) H_{i}^{j} e(\beta),
$$

where $g \in S_{1}, e \in G, i \in I(g, \alpha), z \in P, \lambda>0, j=0, \ldots, n, j \neq i$.

It follows from (14) that

$$
x_{i}^{j-1}-x_{i}^{j}=\left\{\begin{array}{cl}
\left(0, \ldots, 0, z(\lambda) e_{j}(\beta), 0, \ldots, 0\right) & \text { if } j=1, \ldots, n, j \neq i \\
0 & \text { if } j=i .
\end{array}\right.
$$

It is clear that $H_{i}^{0} g=0$ and $x_{i}^{0}(g, e, z, \lambda, \beta)=x+\lambda g$ for all $i \in I(g, \alpha)$.

Definition 7 (see [3]) The discrete gradient of the function $f$ at the point $x \in \mathbb{R}^{n}$ is the vector $\Gamma^{i}(x, g, e, z, \lambda, \beta)=\left(\Gamma_{1}^{i}, \ldots, \Gamma_{n}^{i}\right) \in \mathbb{R}^{n}, g \in S_{1}, i \in I(g, \alpha)$, with the following coordinates:

$$
\begin{gathered}
\Gamma_{j}^{i}=\left[z(\lambda) e_{j}(\beta)\right]^{-1}\left[f\left(x_{i}^{j-1}(g, e, z, \lambda, \beta)\right)-f\left(x_{i}^{j}(g, e, z, \lambda, \beta)\right)\right], \quad j=1, \ldots, n, j \neq i, \\
\Gamma_{i}^{i}=\left(\lambda g_{i}\right)^{-1}\left[f\left(x_{i}^{n}(g, e, z, \lambda, \beta)\right)-f(x)-\sum_{j=1, j \neq i}^{n} \Gamma_{j}^{i}\left(\lambda g_{j}-z(\lambda) e_{j}(\beta)\right)\right] .
\end{gathered}
$$

A more detailed description of the discrete gradient and examples can be found in [4].

Remark 3 It follows from Definition 7 that for the calculation of the discrete gradient $\Gamma^{i}(x, g, e, z, \lambda, \beta), i \in I(g, \alpha)$ we define a sequence of points

$$
x_{i}^{0}, \ldots, x_{i}^{i-1}, x_{i}^{i+1}, \ldots, x_{i}^{n} .
$$

For the calculation of the discrete gradient it is sufficient to evaluate the function $f$ at each point of this sequence.

Remark 4 The discrete gradient is defined with respect to a given direction $g \in S_{1}$. We can see that for the calculation of one discrete gradient we have to calculate $(n+1)$ values of the function $f$ : at the point $x$ and at the points $x_{i}^{j}(g, e, z, \lambda, \beta), j=0, \ldots, n, j \neq i$. For the calculation of the next discrete gradient at the same point with respect to any other direction $g^{1} \in S_{1}$ we have to calculate this function $n$ times, because we have already calculated $f$ at the point $x$.

Remark 5 One can see from (16) that two successive points of the sequence

$$
x_{i}^{0}, \ldots, x_{i}^{i-1}, x_{i}^{i+1}, \ldots, x_{i}^{n}
$$

differ by one coordinate only. More precisely, the point $x^{k}$ can be obtained from the point $x^{k-1}$ by changing only the $k$-th coordinate. 


\subsection{Calculation of the discrete gradients of the function (10)}

We take any point $x \in \mathbb{R}^{n}$ and any direction $g \in S_{1}$. Remark 3 implies that for the calculation of the discrete gradient of $f$ at $x$ with respect to the direction $g$ first we have to define the sequence

$$
x_{i}^{0}, \ldots, x_{i}^{i-1}, x_{i}^{i+1}, \ldots, x_{i}^{n} .
$$

It follows from Remark 5 that each new point $x^{p}$ differs from $x^{p-1}$ by one coordinate only. In order to calculate the discrete gradient we have to evaluate the function $f$ at all of these points.

The functions $f_{i j k}$ are partially separable and they can be represented as

$$
f_{i j k}(x)=\sum_{t=1}^{M_{i j k}} f_{i j k}^{t}\left(U_{i j k t} x\right) .
$$

We will call $f_{i j k}^{t}$ term functions. The total number of these functions is

$$
N_{0}=\sum_{i=1}^{M} \sum_{j \in J_{i}} \sum_{k \in K_{j}} M_{i j k} .
$$

For one evaluation of the function $f$ we have to compute these functions $N_{0}$ times. Since for one evaluation of the discrete gradient we compute $n+1$ times the function $f$, the total number of computation of term functions for one evaluation of the discrete gradient is

$$
N_{t}=(n+1) N_{0} .
$$

For $p \in\{1, \ldots, n\}$ we introduce

$$
\begin{aligned}
& Q_{p}^{i j k}=\left\{t \in\left\{1, \ldots, M_{i j k}\right\}: U_{i j k t}^{p p}=1\right\}, \\
& \bar{Q}_{p}^{i j k}=\left\{t \in\left\{1, \ldots, M_{i j k}\right\}: U_{i j k t}^{p p}=0\right\} .
\end{aligned}
$$

It is clear that $M_{i j k}=\operatorname{card}\left(Q_{p}^{i j k}\right)+\operatorname{card}\left(\bar{Q}_{p}^{i j k}\right)$. One can assume that $\operatorname{card}\left(Q_{p}^{i j k}\right) \ll$ $\operatorname{card}\left(\bar{Q}_{p}^{i j k}\right)$. For example, if all functions $f_{i j k}$ are $l$-chained then

$$
\operatorname{card}\left(Q_{p}^{i j k}\right) \leq l \text { and } \operatorname{card}\left(\bar{Q}_{p}^{i j k}\right) \geq n-l-1 .
$$

If these functions are separable then

$$
\operatorname{card}\left(Q_{p}^{i j k}\right)=1 \text { and } \operatorname{card}\left(\bar{Q}_{p}^{i j k}\right)=n-1 .
$$

Then the function $f_{i j k}$ can be calculated at the point $x^{p}$ using the following simplified scheme:

$$
f_{i j k}\left(x^{p}\right)=\sum_{t \in Q_{p}^{i j k}} f_{i j k}^{t}\left(U_{i j k t} x^{p}\right)+\sum_{t \in \bar{Q}_{p}^{i j k}} f_{i j k}^{t}\left(U_{i j k t} x^{p-1}\right)
$$


that is we compute only functions $f_{i j k}^{t}, t \in Q_{p}^{i j k}$ at the point $x^{p}$ and all other functions remain the same as at the point $x^{p-1}$. Thus in order to calculate the function $f$ at the point $x^{p}$ we compute

$$
N_{s}=\sum_{i=1}^{M} \sum_{j \in J_{i}} \sum_{k \in K_{j}} \operatorname{card}\left(Q_{p}^{i j k}\right)
$$

times the term functions at this point. Since $\operatorname{card}\left(Q_{p}^{i j k}\right) \ll M_{i j k}$ one can expect that $N_{s} \ll N_{0}$.

If all functions $f_{i j k}, i=1, \ldots, M, j \in J_{i}, k \in K_{j}$ are $l$-chained then

$$
N_{s} \leq l \sum_{i=1}^{M} \sum_{j \in J_{i}} \operatorname{card}\left(K_{j}\right) .
$$

If all these functions are separable then

$$
N_{s}=\sum_{i=1}^{M} \sum_{j \in J_{i}} \operatorname{card}\left(K_{j}\right) .
$$

Thus in order to compute one discrete gradient at the point $x$ with respect to the direction $g \in S_{1}$ we have to compute the function $f$ at the points $x$ and $x+\lambda g$ using formula (10) and at all other points $x_{i}^{p}, p=1, \ldots, n, p \neq i$ it can be computed using simplified scheme (17). In this case the total number of computation of term functions is

$$
N_{t s}=2 N_{0}+(n-1) N_{s}
$$

which is significantly less than $N_{t}$ when $n$ is large.

Now we consider one special case of functions (10).

\subsubsection{Functions represented as a sum of minimum functions}

We consider the following functions:

$$
f(x)=\sum_{i=1}^{M} \min _{k \in \bar{K}} f_{i k}\left(x^{k}\right)
$$

where $\bar{K}=\{1, \ldots, K\}, x^{k} \in \mathbb{R}^{n}, x=\left(x^{1}, \ldots, x^{K}\right) \in \mathbb{R}^{K \times n}$ and the functions $f_{i k}$ are separable

$$
f_{i k}(x)=\sum_{j=1}^{n} f_{i j k}\left(x_{j}^{k}\right) .
$$

The function (18) can be derived from the function (10) when

$$
J_{i}=\{1\}, \quad i=1, \ldots, M, \quad K_{j}=\{1, \ldots, K\} .
$$


In order to calculate one discrete gradient of the function (18) we have to evaluate $M K(n+$ 1 ) times the functions $f_{i j k}$. However the use of the simplified scheme reduces this number to $2 M K+n-1$.

One of the special cases of functions (18) is the cluster function (4). This function can be rewritten as follows

$$
f(x)=\sum_{i=1}^{M} \min _{k=1, \ldots, K}\left\|x^{k}-a^{i}\right\|^{2}, \quad x=\left(x^{1}, \ldots, x^{K}\right) \in \mathbb{R}^{K \times n} .
$$

Here

$$
f_{i k}\left(x^{k}\right)=\left\|x^{k}-a^{i}\right\|^{2} \quad \text { and } \quad f_{i j k}\left(x_{j}^{k}\right)=\left(x_{j}^{k}-a_{j}^{i}\right)^{2} .
$$

For the computation of one discrete gradient without using simplified scheme we have to compute $M K(n+1)$ the very simple functions $f_{i j k}$, however the use of the simplified scheme allows one to reduce this number to $2 M K+n-1$. Since in the cluster analysis the number $M$ is large we can assume that $M K \gg n$ and therefore

$$
\frac{M K(n+1)}{2 M K+n-1} \approx \frac{n+1}{2} .
$$

If $n$ is large then we can significantly reduce computational efforts using the simplified scheme.

\subsubsection{Discrete gradient method}

In this subsection we briefly describe the discrete gradient method. A more detailed description of this method can be found in $[4,6]$.

We consider the following unconstrained minimization problem:

$$
\text { minimize } f(x) \text { subject to } x \in \mathbb{R}^{n}
$$

where the function $f$ is assumed to be semismooth. An important step in the discrete gradient method is the calculation of a descent direction of the objective function $f$.

Let $z \in P, \lambda>0, \beta \in(0,1]$, the number $c \in(0,1)$ and a small enough number $\delta>0$ be given.

Algorithm 1 An algorithm for the computation of the descent direction.

Step 1. Choose any $g^{1} \in S_{1}, e \in G, i \in I\left(g^{1}, \alpha\right)$ and compute a discrete gradient $v^{1}=$ $\Gamma^{i}\left(x, g^{1}, e, z, \lambda, \beta\right)$. Set $\bar{D}_{1}(x)=\left\{v^{1}\right\}$ and $k=1$.

Step 2. Calculate the vector $\left\|w^{k}\right\|^{2}=\min \left\{\|w\|^{2}: w \in \bar{D}_{k}(x)\right\}$. If

$$
\left\|w^{k}\right\| \leq \delta
$$

then stop. Otherwise go to Step 3.

Step 3. Calculate the search direction by $g^{k+1}=-\left\|w^{k}\right\|^{-1} w^{k}$. 
Step 4. If

$$
f\left(x+\lambda g^{k+1}\right)-f(x) \leq-c \lambda\left\|w^{k}\right\|,
$$

then stop. Otherwise go to Step 5.

Step 5. Calculate a discrete gradient

$$
v^{k+1}=\Gamma^{i}\left(x, g^{k+1}, e, z, \lambda, \beta\right), i \in I\left(g^{k+1}, \alpha\right),
$$

construct the set $\bar{D}_{k+1}(x)=\operatorname{co}\left\{\bar{D}_{k}(x) \bigcup\left\{v^{k+1}\right\}\right\}$, set $k=k+1$ and go to Step 2 .

Explanations to Algorithm 1. In Step 1 we calculate the first discrete gradient. The distance between the convex hull of all calculated discrete gradients and the origin is calculated in Step 2. If this distance is less than the tolerance $\delta>0$ then we accept the point $x$ as an approximate stationary point (Step 2), otherwise we calculate another search direction in Step 3. In Step 4 we check whether this direction is a descent direction. If it is we stop and the descent direction has been calculated, otherwise we calculate another discrete gradient with respect to this direction in Step 5 and add it to the set $\bar{D}_{k}$.

It is proved that Algorithm 1 is terminating (see $[4,6]$ ).

Let numbers $c_{1} \in(0,1), c_{2} \in\left(0, c_{1}\right]$ be given.

Algorithm 2 Discrete gradient method

Step 1. Choose any starting point $x^{0} \in \mathbb{R}^{n}$ and set $k=0$.

Step 2. Set $s=0$ and $x_{s}^{k}=x^{k}$.

Step 3. Apply Algorithm 1 for the calculation of the descent direction at $x=x_{s}^{k}, \delta=$ $\delta_{k}, z=z_{k}, \lambda=\lambda_{k}, \beta=\beta_{k}, c=c_{1}$. This algorithm terminates after a finite number of iterations $m>0$. As a result we get the set $D_{m}\left(x_{s}^{k}\right)$ and an element $v_{s}^{k}$ such that

$$
\left\|v_{s}^{k}\right\|^{2}=\min \left\{\|v\|^{2}: v \in \bar{D}_{m}\left(x_{s}^{k}\right)\right\} .
$$

Furthermore either $\left\|v_{s}^{k}\right\| \leq \delta_{k}$ or for the search direction $g_{s}^{k}=-\left\|v_{s}^{k}\right\|^{-1} v_{s}^{k}$

$$
f\left(x_{s}^{k}+\lambda_{k} g_{s}^{k}\right)-f\left(x_{s}^{k}\right) \leq-c_{1} \lambda_{k}\left\|v_{s}^{k}\right\| .
$$

Step 4. If

$$
\left\|v_{s}^{k}\right\| \leq \delta_{k}
$$

then set $x^{k+1}=x_{s}^{k}, k=k+1$ and go to Step 2. Otherwise go to Step 5 .

Step 5. Construct the following iteration $x_{s+1}^{k}=x_{s}^{k}+\sigma_{s} g_{s}^{k}$, where $\sigma_{s}$ is defined as follows

$$
\sigma_{s}=\operatorname{argmax}\left\{\sigma \geq 0: f\left(x_{s}^{k}+\sigma g_{s}^{k}\right)-f\left(x_{s}^{k}\right) \leq-c_{2} \sigma\left\|v_{s}^{k}\right\|\right\} .
$$

Step 6. Set $s=s+1$ and go to Step 3 .

The convergence of the discrete gradient method is studied in $[4,6]$. 


\section{Results of numerical experiments}

A number of numerical experiments have been carried out using large scale nonsmooth optimization problems.

\subsection{Test problems}

The following test problems have been used in numerical experiments. The description of chained functions can be also found in [18, 23, 24]. We consider unconstrained minimization problems. Below $f_{*}$ stands for the minimum value of a function $f$.

\subsubsection{Piecewise chained problems}

\section{Problem 1 Chained LQ function}

$$
f(x)=\sum_{i=1}^{n-1} \max \left\{-x_{i}-x_{i+1},-x_{i}-x_{i+1}+\left(x_{i}^{2}+x_{i+1}^{2}-1\right)\right\}, \quad f_{*}=-(n-1) \sqrt{2} .
$$

\section{Problem 2 Chained CB3 I function}

$$
f(x)=\sum_{i=1}^{n-1} \max \left\{x_{i}^{4}+x_{i+1}^{2},\left(2-x_{i}\right)^{2}+\left(2-x_{i+1}\right)^{2}, 2 e^{-x_{i}+x_{i+1}}\right\}, \quad f_{*}=2(n-1) .
$$

\section{Problem 3 Chained CB3 II function}

$$
\begin{gathered}
f(x)=\max \left\{\sum_{i=1}^{n-1}\left(x_{i}^{4}+x_{i+1}^{2}\right), \sum_{i=1}^{n-1}\left(\left(2-x_{i}\right)^{2}+\left(2-x_{i+1}\right)^{2}\right), 2 \sum_{i=1}^{n-1} e^{-x_{i}+x_{i+1}}\right\}, \\
f_{*}=2(n-1) .
\end{gathered}
$$

Problem 4 Nonsmooth generalization of Brown function 2

$$
f(x)=\sum_{i=1}^{n-1}\left(\left|x_{i}\right|^{x_{i+1}^{2}+1}+\left|x_{i+1}\right|^{x_{i}^{2}+1}\right), \quad f_{*}=0 .
$$

\section{Problem 5 Chained Mifflin 2 function}

$$
f(x)=\sum_{i=1}^{n-1}\left(-x_{i}+2\left(x_{i}^{2}-x_{i+1}^{2}-1\right)+1.75\left|x_{i}^{2}+x_{i+1}^{2}-1\right|\right), \quad f_{*} \quad \text { varies. }
$$


Problem 6 Chained Crescent I function

$$
\begin{gathered}
f(x)=\max \left\{\sum_{i=1}^{n-1}\left(x_{i}^{2}+\left(x_{i+1}-1\right)^{2}+x_{i+1}-1\right), \sum_{i=1}^{n-1}\left(-x_{i}^{2}-\left(x_{i+1}-1\right)^{2}+x_{i+1}+1\right)\right\}, \\
f_{*}=0 .
\end{gathered}
$$

Problem 7 Chained Crescent II function

$$
f(x)=\sum_{i=1}^{n-1} \max \left\{x_{i}^{2}+\left(x_{i+1}-1\right)^{2}+x_{i+1}-1,-x_{i}^{2}-\left(x_{i+1}-1\right)^{2}+x_{i+1}+1\right\}, \quad f_{*}=0 .
$$

Problem 8 Chained Wood function

$$
\begin{gathered}
f(x)=\sum_{j=1}^{k}\left[100\left(x_{2 j-1}^{2}-x_{2 j}\right)^{2}+\left(x_{2 j-1}-1\right)^{2}+90\left(x_{2 j+1}^{2}-x_{2 j+2}\right)^{2}+\left(x_{2 j+1}-1\right)^{2}\right. \\
\left.+10\left(x_{2 j}+x_{2 j+1}-2\right)^{2}+\left(x_{2 j}-x_{2 j+2}\right)^{2} / 10\right] \\
k=(n-2) / 2, \quad f_{*}=0 .
\end{gathered}
$$

Problem 9 Chained Powell singular function

$$
\begin{gathered}
f(x)=\sum_{j=1}^{k}\left[\left(x_{2 j-1}+10 x_{2 j}\right)^{2}+5\left(x_{2 j+1}-x_{2 j+2}\right)^{2}+\left(x_{2 j}-2 x_{2 j+1}\right)^{4}+10\left(x_{2 j-1}-x_{2 j+2}\right)^{4}\right], \\
k=(n-2) / 2, \quad f_{*}=0 .
\end{gathered}
$$

\subsubsection{Piecewise partially separable problems}

\section{Problem 10 PPSF CB3 I function}

$$
f(x)=\sum_{i=1}^{n} \max \left\{x_{i}^{4}+x_{1}^{2},\left(2-x_{i}\right)^{2}+\left(2-x_{1}\right)^{2}, 2 e^{-x_{i}+x_{1}}\right\}, \quad f_{*}=2 n .
$$

\section{Problem 11 PPSF CB3 II function}

$$
f(x)=\max \left\{\sum_{i=1}^{n}\left(x_{i}^{4}+x_{1}^{2}\right), \sum_{i=1}^{n}\left(\left(2-x_{i}\right)^{2}+\left(2-x_{1}\right)^{2}\right), 2 \sum_{i=1}^{n}\left(e^{-x_{i}+x_{1}}\right)\right\}, \quad f_{*}=2 n .
$$




\section{Problem 12 PPSF Nonsmooth generalization of Brown function 2}

$$
f(x)=\sum_{i=1}^{n}\left(\left|x_{i}\right|^{x_{1}^{2}+1}+\left|x_{1}\right|^{x_{i}^{2}+1}\right), \quad f_{*}=0 .
$$

\section{Problem 13 PPSF Broyden function}

$$
f(x)=\sum_{i=1}^{n}\left|\left(3-2 x_{i}\right) x_{i}-x_{1}-x_{2}+1\right|^{7 / 3}, \quad f_{*}=0 .
$$

It should be noted that Problems 8, 9 have smooth objective functions. The objective functions in Problems 10-13 are piecewise partially separable and they are modification of corresponding test functions from [23].

The code has been written in $\mathrm{C}++$ and numerical experiments have been carried out on a PC Intel Pentium 4, 1.6 MHz. Their results are presented in Tables 1-3. In these tables we use the following notations:

- $n$ is the number of variables;

- $t$ the CPU time in seconds;

- $N_{f}$ the number of evaluations of term functions when the simplified scheme is applied;

- $N_{S}$ the number of objective function evaluations when the simplified scheme is applied;

- $N_{g}$ the number of objective function evaluations without application of the simplified scheme;

- $x^{0}$ and $x^{*}$ are the initial point and the minimizer, respectively.

We consider that starting from the point $x^{0}$ the algorithm succeeds if for the final point $\bar{x}$ the inequality

$$
\frac{f(\bar{x})-f_{*}}{\left|f_{*}\right|+1}<\epsilon
$$

is true. Otherwise we say that it fails. Here the tolerance $\epsilon=10^{-4}$.

In the numerical experiments for each problem and $n$ we ran the algorithm starting from 100 randomly chosen points. In the tables we present average values of $t, N_{f}, N_{S}$ and $N_{g} / N_{S}$. In the column "Failed" we present the number of failures of the algorithm. We also present the minimum and maximum values of the difference $f\left(x^{0}\right)-f\left(x^{*}\right)$ in order to demonstrate how far the initial points are from the solution.

Figures 1 and 3 show the dependence of $N_{S}$ on the number of variables $n$ for piecewise chained and piecewise partially separable functions, respectively. Figures 2 and 4 show the dependence of $N_{g} / N_{S}$ on the number of variables $n$ for these functions. 


\begin{tabular}{|c|c|c|c|c|c|c|c|c|}
\hline & \multirow[b]{2}{*}{$n$} & \multirow[b]{2}{*}{$t$} & \multirow[b]{2}{*}{$N_{f}$} & \multirow[b]{2}{*}{$N_{S}$} & \multirow[b]{2}{*}{$N_{g} / N_{S}$} & \multirow[b]{2}{*}{ Failed } & \multicolumn{2}{|c|}{$f\left(x_{0}\right)-f\left(x^{*}\right)$} \\
\hline & & & & & & & $\min$ & $\max$ \\
\hline \multirow{9}{*}{$\begin{array}{l}\text { Chained } \\
\text { LQ }\end{array}$} & 2000 & 44.30 & $3.23 \mathrm{e} 7$ & $1.62 \mathrm{e} 4$ & 1800.0 & 0 & $1.27 \mathrm{e} 05$ & $1.43 \mathrm{e} 05$ \\
\hline & 1500 & 23.60 & $1.84 \mathrm{e} 7$ & $1.23 \mathrm{e} 4$ & 1310.0 & 0 & $9.34 \mathrm{e} 04$ & $1.07 \mathrm{e} 05$ \\
\hline & 1000 & 11.40 & $9.28 \mathrm{e} 6$ & $9.29 \mathrm{e} 3$ & 859.0 & 0 & $6.32 \mathrm{e} 04$ & $7.27 \mathrm{e} 04$ \\
\hline & 800 & 8.31 & $6.66 \mathrm{e} 6$ & $8.34 \mathrm{e} 3$ & 686.0 & 0 & $4.84 \mathrm{e} 04$ & $5.80 \mathrm{e} 04$ \\
\hline & 500 & 5.18 & $4.10 \mathrm{e} 6$ & $8.21 \mathrm{e} 3$ & 416.0 & 0 & $2.99 \mathrm{e} 04$ & $3.76 \mathrm{e} 04$ \\
\hline & 300 & 3.26 & $2.38 \mathrm{e} 6$ & $7.95 \mathrm{e} 3$ & 244.0 & 0 & $1.79 \mathrm{e} 04$ & $2.22 \mathrm{e} 04$ \\
\hline & 100 & 1.76 & $7.58 \mathrm{e} 5$ & $7.66 \mathrm{e} 3$ & 79.0 & 0 & $5.24 \mathrm{e} 03$ & $8.65 \mathrm{e} 03$ \\
\hline & 50 & 1.31 & $3.02 \mathrm{e} 5$ & $6.17 \mathrm{e} 3$ & 39.9 & 0 & $2.33 \mathrm{e} 03$ & $4.47 \mathrm{e} 03$ \\
\hline & 10 & 0.34 & $1.10 \mathrm{e} 4$ & $1.22 \mathrm{e} 3$ & 5.6 & 0 & $2.54 \mathrm{e} 02$ & $1.00 \mathrm{e} 03$ \\
\hline \multirow{9}{*}{$\begin{array}{l}\text { Chained } \\
\text { CB3 I }\end{array}$} & 2000 & 81.20 & $3.26 \mathrm{e} 7$ & $1.63 \mathrm{e} 4$ & 1380.0 & 0 & $2.73 \mathrm{e} 09$ & $7.80 \mathrm{e} 09$ \\
\hline & 1500 & 49.00 & $2.13 \mathrm{e} 7$ & $1.42 \mathrm{e} 4$ & 963.0 & 0 & $1.86 \mathrm{e} 09$ & $6.71 \mathrm{e} 09$ \\
\hline & 1000 & 25.60 & $1.14 \mathrm{e} 7$ & $1.14 \mathrm{e} 4$ & 633.0 & 0 & $9.85 \mathrm{e} 08$ & $4.39 \mathrm{e} 09$ \\
\hline & 800 & 18.70 & $8.29 \mathrm{e} 6$ & $1.04 \mathrm{e} 4$ & 511.0 & 0 & $2.28 \mathrm{e} 08$ & $4.23 \mathrm{e} 09$ \\
\hline & 500 & 9.25 & $4.20 \mathrm{e} 6$ & $8.42 \mathrm{e} 3$ & 309.0 & 0 & $3.29 \mathrm{e} 08$ & $3.13 \mathrm{e} 09$ \\
\hline & 300 & 3.56 & $1.81 \mathrm{e} 6$ & $6.04 \mathrm{e} 3$ & 163.0 & 0 & $1.17 \mathrm{e} 08$ & $2.26 \mathrm{e} 09$ \\
\hline & 100 & 0.75 & $3.93 \mathrm{e} 5$ & $3.97 \mathrm{e} 3$ & 49.2 & 0 & $2.95 \mathrm{e} 06$ & $9.50 \mathrm{e} 08$ \\
\hline & 50 & 0.37 & $1.69 \mathrm{e} 5$ & $3.46 \mathrm{e} 3$ & 24.6 & 0 & $9.48 \mathrm{e} 04$ & $6.97 \mathrm{e} 08$ \\
\hline & 10 & 0.03 & $1.44 \mathrm{e} 4$ & $1.60 \mathrm{e} 3$ & 3.0 & 0 & $3.96 \mathrm{e} 03$ & $3.55 \mathrm{e} 08$ \\
\hline \multirow{9}{*}{$\begin{array}{l}\text { Chained } \\
\text { CB3II }\end{array}$} & 2000 & 40.20 & $2.07 \mathrm{e} 7$ & $1.03 \mathrm{e} 4$ & 1250.0 & 0 & $2.92 \mathrm{e} 09$ & $7.65 \mathrm{e} 09$ \\
\hline & 1500 & 18.2 & $1.06 \mathrm{e} 7$ & $7.04 \mathrm{e} 3$ & 806.0 & 0 & $2.01 \mathrm{e} 09$ & $5.76 \mathrm{e} 09$ \\
\hline & 1000 & 8.22 & $5.19 \mathrm{e} 6$ & $5.19 \mathrm{e} 3$ & 488.0 & 0 & $9.61 \mathrm{e} 08$ & $4.42 \mathrm{e} 09$ \\
\hline & 800 & 5.72 & $3.62 \mathrm{e} 6$ & $4.53 \mathrm{e} 3$ & 385.0 & 0 & $7.16 \mathrm{e} 08$ & $3.84 \mathrm{e} 09$ \\
\hline & 500 & 2.93 & $1.87 \mathrm{e} 6$ & $3.76 \mathrm{e} 3$ & 243.0 & 0 & $3.73 \mathrm{e} 08$ & $2.99 \mathrm{e} 09$ \\
\hline & 300 & 1.50 & $9.44 \mathrm{e} 5$ & $3.16 \mathrm{e} 3$ & 150.0 & 0 & $5.76 \mathrm{e} 07$ & $2.39 \mathrm{e} 09$ \\
\hline & 100 & 0.50 & $2.37 \mathrm{e} 5$ & $2.39 \mathrm{e} 3$ & 54.0 & 0 & $1.05 \mathrm{e} 07$ & $1.40 \mathrm{e} 09$ \\
\hline & 50 & 0.29 & $9.83 \mathrm{e} 4$ & $2.01 \mathrm{e} 3$ & 26.2 & 0 & $1.19 \mathrm{e} 05$ & $1.06 \mathrm{e} 09$ \\
\hline & 10 & 0.02 & $1.03 \mathrm{e} 4$ & $1.15 \mathrm{e} 3$ & 3.9 & 0 & $4.38 \mathrm{e} 03$ & $2.42 \mathrm{e} 08$ \\
\hline \multirow{9}{*}{$\begin{array}{l}\text { Chained } \\
\text { generalised } \\
\text { Brown } 2\end{array}$} & 2000 & 76.60 & $1.79 \mathrm{e} 7$ & $8.94 \mathrm{e} 3$ & 1850.0 & 5 & $8.86 \mathrm{e} 02$ & $9.37 \mathrm{e} 02$ \\
\hline & 1500 & 32.30 & $7.76 \mathrm{e} 6$ & $5.18 \mathrm{e} 3$ & 1360.0 & 0 & $6.56 \mathrm{e} 02$ & $7.09 \mathrm{e} 02$ \\
\hline & 1000 & 15.30 & $3.83 \mathrm{e} 6$ & $3.84 \mathrm{e} 3$ & 874.0 & 0 & $4.35 \mathrm{e} 02$ & $4.77 \mathrm{e} 02$ \\
\hline & 800 & 9.75 & $2.50 \mathrm{e} 6$ & $3.12 \mathrm{e} 3$ & 689.0 & 0 & $3.49 \mathrm{e} 02$ & $3.77 \mathrm{e} 02$ \\
\hline & 500 & 4.91 & $1.15 \mathrm{e} 6$ & $2.30 \mathrm{e} 3$ & 425.0 & 0 & $2.14 \mathrm{e} 02$ & $2.42 \mathrm{e} 02$ \\
\hline & 300 & 3.24 & $5.78 \mathrm{e} 5$ & $1.93 \mathrm{e} 3$ & 250.0 & 0 & $1.28 \mathrm{e} 02$ & $1.45 \mathrm{e} 02$ \\
\hline & 100 & 1.57 & $1.38 \mathrm{e} 5$ & $1.39 \mathrm{e} 3$ & 82.1 & 0 & $4.02 \mathrm{e} 01$ & $4.99 \mathrm{e} 01$ \\
\hline & 50 & 2.48 & $5.56 \mathrm{e} 4$ & $1.14 \mathrm{e} 3$ & 40.3 & 0 & $1.86 \mathrm{e} 01$ & $2.64 \mathrm{e} 01$ \\
\hline & 10 & 0.03 & $4.53 \mathrm{e} 3$ & $5.03 \mathrm{e} 2$ & 6.6 & 0 & $2.39 \mathrm{e} 00$ & $5.87 \mathrm{e} 00$ \\
\hline
\end{tabular}

Table 1: Results for piecewise chained functions 


\begin{tabular}{|c|c|c|c|c|c|c|c|c|}
\hline & \multirow[b]{2}{*}{$n$} & \multirow[b]{2}{*}{$t$} & \multirow[b]{2}{*}{$N_{f}$} & \multirow[b]{2}{*}{$N_{S}$} & \multirow[b]{2}{*}{$N_{g} / N_{S}$} & \multirow[b]{2}{*}{ Failed } & \multicolumn{2}{|c|}{$f\left(x_{0}\right)-f\left(x^{*}\right)$} \\
\hline & & & & & & & $\min$ & $\max$ \\
\hline Chained & 2000 & 10.70 & $8.84 \mathrm{e} 6$ & $4.42 \mathrm{e} 3$ & 1840.0 & 5 & $1.27 \mathrm{e} 05$ & $1.43 \mathrm{e} 05$ \\
\hline \multirow{8}{*}{ Crescent I } & 1500 & 5.51 & $4.72 \mathrm{e} 6$ & $3.15 \mathrm{e} 3$ & 1350.0 & 3 & $9.48 \mathrm{e} 04$ & $1.09 \mathrm{e} 05$ \\
\hline & 1000 & 2.45 & $2.23 \mathrm{e} 6$ & $2.23 \mathrm{e} 3$ & 875.0 & 0 & $6.26 \mathrm{e} 04$ & $7.05 \mathrm{e} 04$ \\
\hline & 800 & 1.71 & $1.56 \mathrm{e} 6$ & $1.95 \mathrm{e} 3$ & 692.0 & 0 & $4.87 \mathrm{e} 04$ & $5.72 \mathrm{e} 04$ \\
\hline & 500 & 0.99 & $8.62 \mathrm{e} 5$ & $1.73 \mathrm{e} 3$ & 428.0 & 0 & $2.93 \mathrm{e} 04$ & $3.60 \mathrm{e} 04$ \\
\hline & 300 & 0.62 & $4.54 \mathrm{e} 5$ & $1.52 \mathrm{e} 3$ & 256.0 & 0 & $1.78 \mathrm{e} 04$ & $2.30 \mathrm{e} 04$ \\
\hline & 100 & 0.30 & $1.19 \mathrm{e} 5$ & $1.20 \mathrm{e} 3$ & 82.9 & 0 & $5.37 \mathrm{e} 03$ & $8.04 \mathrm{e} 03$ \\
\hline & 50 & 0.23 & $5.18 \mathrm{e} 4$ & $1.06 \mathrm{e} 3$ & 40.5 & 0 & $2.33 \mathrm{e} 03$ & $4.47 \mathrm{e} 03$ \\
\hline & 10 & 0.03 & $5.78 \mathrm{e} 3$ & $6.42 \mathrm{e} 2$ & 6.7 & 0 & $2.83 \mathrm{e} 02$ & $9.95 \mathrm{e} 02$ \\
\hline Chained & 2000 & 25.80 & $2.15 \mathrm{e} 7$ & $1.08 \mathrm{e} 4$ & 1760.0 & 100 & $1.27 \mathrm{e} 05$ & $1.41 \mathrm{e} 05$ \\
\hline \multirow[t]{8}{*}{ Crescent II } & 1500 & 11.20 & $1.07 \mathrm{e} 7$ & $7.17 \mathrm{e} 3$ & 1250.0 & 99 & $9.40 \mathrm{e} 04$ & $1.06 \mathrm{e} 05$ \\
\hline & 1000 & 4.45 & $5.14 \mathrm{e} 6$ & $5.15 \mathrm{e} 3$ & 779.0 & 100 & $6.20 \mathrm{e} 04$ & $7.23 \mathrm{e} 04$ \\
\hline & 800 & 2.83 & $3.62 \mathrm{e} 6$ & $4.53 \mathrm{e} 3$ & 606.0 & 98 & $4.96 \mathrm{e} 04$ & $5.86 \mathrm{e} 04$ \\
\hline & 500 & 1.30 & $1.84 \mathrm{e} 6$ & $3.69 \mathrm{e} 3$ & 361.0 & 99 & $3.01 \mathrm{e} 04$ & $3.64 \mathrm{e} 04$ \\
\hline & 300 & 0.70 & $8.95 \mathrm{e} 5$ & $2.99 \mathrm{e} 3$ & 207.0 & 97 & $1.76 \mathrm{e} 04$ & $2.25 \mathrm{e} 04$ \\
\hline & 100 & 2.48 & $2.53 \mathrm{e} 5$ & $2.55 \mathrm{e} 3$ & 71.2 & 61 & $5.11 \mathrm{e} 03$ & $8.56 \mathrm{e} 03$ \\
\hline & 50 & 0.50 & $1.25 \mathrm{e} 5$ & $2.55 \mathrm{e} 3$ & 39.1 & 0 & $2.31 \mathrm{e} 03$ & $4.46 \mathrm{e} 03$ \\
\hline & 10 & 0.26 & $7.51 \mathrm{e} 3$ & $8.34 \mathrm{e} 2$ & 6.0 & 0 & $2.61 \mathrm{e} 02$ & $9.23 \mathrm{e} 02$ \\
\hline \multirow{9}{*}{$\begin{array}{l}\text { Chained } \\
\text { Mifflin }\end{array}$} & 2000 & 85.20 & $7.18 \mathrm{e} 7$ & $3.59 \mathrm{e} 4$ & 1700.0 & 0 & $4.69 \mathrm{e} 05$ & $5.16 \mathrm{e} 05$ \\
\hline & 1500 & 63.50 & $6.73 \mathrm{e} 7$ & $4.49 \mathrm{e} 4$ & 1140.0 & 0 & $3.48 \mathrm{e} 05$ & $3.91 \mathrm{e} 05$ \\
\hline & 1000 & 22.50 & $2.83 \mathrm{e} 7$ & $2.83 \mathrm{e} 4$ & 708.0 & 0 & $2.29 \mathrm{e} 05$ & $2.68 \mathrm{e} 05$ \\
\hline & 800 & 14.00 & $1.83 \mathrm{e} 7$ & $2.29 \mathrm{e} 4$ & 549.0 & 0 & $1.84 \mathrm{e} 05$ & $2.11 \mathrm{e} 05$ \\
\hline & 500 & 6.13 & $8.08 \mathrm{e} 6$ & $1.62 \mathrm{e} 4$ & 331.0 & 0 & $1.13 \mathrm{e} 05$ & $1.33 \mathrm{e} 05$ \\
\hline & 300 & 3.15 & $3.73 \mathrm{e} 6$ & $1.25 \mathrm{e} 4$ & 198.0 & 0 & $6.61 \mathrm{e} 04$ & $8.37 \mathrm{e} 04$ \\
\hline & 100 & 1.60 & $7.97 \mathrm{e} 5$ & $8.05 \mathrm{e} 3$ & 75.0 & 0 & $1.76 \mathrm{e} 04$ & $3.10 \mathrm{e} 04$ \\
\hline & 50 & 1.65 & $3.37 \mathrm{e} 5$ & $6.88 \mathrm{e} 3$ & 40.0 & 0 & $8.59 \mathrm{e} 03$ & $1.81 \mathrm{e} 04$ \\
\hline & 10 & 0.91 & $1.39 \mathrm{e} 4$ & $1.54 \mathrm{e} 3$ & 6.0 & 0 & $9.15 \mathrm{e} 02$ & $3.84 \mathrm{e} 03$ \\
\hline \multirow{9}{*}{$\begin{array}{l}\text { Chained } \\
\text { Wood }\end{array}$} & 2000 & 62.00 & $4.27 \mathrm{e} 7$ & $2.14 \mathrm{e} 4$ & 1290.0 & 0 & $3.31 \mathrm{e} 08$ & $3.86 \mathrm{e} 08$ \\
\hline & 1500 & 34.20 & $2.44 \mathrm{e} 7$ & $1.63 \mathrm{e} 4$ & 844.0 & 0 & $2.49 \mathrm{e} 08$ & $3.05 \mathrm{e} 08$ \\
\hline & 1000 & 19.20 & $1.26 \mathrm{e} 7$ & $1.26 \mathrm{e} 4$ & 466.0 & 0 & $1.62 \mathrm{e} 08$ & $1.99 \mathrm{e} 08$ \\
\hline & 800 & 14.00 & $8.77 \mathrm{e} 6$ & $1.10 \mathrm{e} 4$ & 345.0 & 0 & $1.22 \mathrm{e} 08$ & $1.72 \mathrm{e} 08$ \\
\hline & 500 & 13.20 & $4.39 \mathrm{e} 6$ & $8.79 \mathrm{e} 3$ & 201.0 & 0 & $7.79 \mathrm{e} 07$ & $1.11 \mathrm{e} 08$ \\
\hline & 300 & 10.40 & $2.25 \mathrm{e} 6$ & $7.51 \mathrm{e} 3$ & 117.0 & 0 & $4.20 \mathrm{e} 07$ & $6.59 \mathrm{e} 07$ \\
\hline & 100 & 11.40 & $6.19 \mathrm{e} 5$ & $6.25 \mathrm{e} 3$ & 40.6 & 0 & $1.08 \mathrm{e} 07$ & $2.77 \mathrm{e} 07$ \\
\hline & 50 & 11.10 & $2.73 \mathrm{e} 5$ & $5.57 \mathrm{e} 3$ & 22.7 & 0 & $4.35 \mathrm{e} 06$ & $1.28 \mathrm{e} 07$ \\
\hline & 10 & 0.29 & $2.75 \mathrm{e} 4$ & $3.05 \mathrm{e} 3$ & 3.7 & 0 & $5.70 \mathrm{e} 04$ & $3.18 \mathrm{e} 06$ \\
\hline \multirow{9}{*}{$\begin{array}{l}\text { Chained } \\
\text { Powell } \\
\text { singular }\end{array}$} & 2000 & 24.10 & $1.53 \mathrm{e} 7$ & $7.64 \mathrm{e} 3$ & 1040.0 & 0 & $1.48 \mathrm{e} 08$ & $1.88 \mathrm{e} 08$ \\
\hline & 1500 & 13.90 & $9.13 \mathrm{e} 6$ & $6.09 \mathrm{e} 3$ & 658.0 & 0 & $1.10 \mathrm{e} 08$ & $1.52 \mathrm{e} 08$ \\
\hline & 1000 & 8.89 & $5.29 \mathrm{e} 6$ & $5.30 \mathrm{e} 3$ & 402.0 & 0 & $7.19 \mathrm{e} 07$ & $1.01 \mathrm{e} 08$ \\
\hline & 800 & 13.00 & $4.13 \mathrm{e} 6$ & $5.17 \mathrm{e} 3$ & 325.0 & 0 & $5.08 \mathrm{e} 07$ & $8.58 \mathrm{e} 07$ \\
\hline & 500 & 6.42 & $2.52 \mathrm{e} 6$ & $5.04 \mathrm{e} 3$ & 219.0 & 0 & $3.36 \mathrm{e} 07$ & $5.68 \mathrm{e} 07$ \\
\hline & 300 & 5.72 & $1.67 \mathrm{e} 6$ & $5.58 \mathrm{e} 3$ & 142.0 & 0 & $1.78 \mathrm{e} 07$ & $3.67 \mathrm{e} 07$ \\
\hline & 100 & 5.69 & $5.40 \mathrm{e} 5$ & $5.45 \mathrm{e} 3$ & 44.4 & 0 & $4.33 \mathrm{e} 06$ & $1.36 \mathrm{e} 07$ \\
\hline & 50 & 5.44 & $2.24 \mathrm{e} 5$ & $4.58 \mathrm{e} 3$ & 21.6 & 0 & $1.26 \mathrm{e} 06$ & $7.62 \mathrm{e} 06$ \\
\hline & 10 & 0.21 & $3.79 \mathrm{e} 4$ & $4.22 \mathrm{e} 3$ & 3.3 & 0 & $2.49 \mathrm{e} 04$ & $1.77 \mathrm{e} 06$ \\
\hline
\end{tabular}

Table 2: Results for piecewise chained functions 


\begin{tabular}{|c|c|c|c|c|c|c|c|c|}
\hline & \multirow[b]{2}{*}{$n$} & \multirow[b]{2}{*}{$t$} & \multirow[b]{2}{*}{$N_{f}$} & \multirow[b]{2}{*}{$N_{S}$} & \multirow[b]{2}{*}{$N_{g} / N_{S}$} & \multirow[b]{2}{*}{ Failed } & \multicolumn{2}{|c|}{$f\left(x_{0}\right)-f\left(x^{*}\right)$} \\
\hline & & & & & & & $\min$ & $\max$ \\
\hline PPSF & 2000 & 51.20 & $3.87 \mathrm{e} 7$ & $1.94 \mathrm{e} 4$ & 854.0 & 0 & $3.82 \mathrm{e} 06$ & $8.07 \mathrm{e} 10$ \\
\hline \multirow[t]{8}{*}{ CB3 I } & 1500 & 27.50 & $2.19 \mathrm{e} 7$ & $1.46 \mathrm{e} 4$ & 605.0 & 0 & $2.80 \mathrm{e} 06$ & $7.70 \mathrm{e} 10$ \\
\hline & 1000 & 12.90 & $1.07 \mathrm{e} 7$ & $1.07 \mathrm{e} 4$ & 384.0 & 0 & $1.88 \mathrm{e} 06$ & $2.93 \mathrm{e} 10$ \\
\hline & 800 & 9.26 & $7.85 \mathrm{e} 6$ & $9.82 \mathrm{e} 3$ & 298.0 & 0 & $1.49 \mathrm{e} 06$ & $3.99 \mathrm{e} 10$ \\
\hline & 500 & 4.93 & $4.26 \mathrm{e} 6$ & $8.53 \mathrm{e} 3$ & 178.0 & 0 & $9.11 \mathrm{e} 05$ & $2.00 \mathrm{e} 10$ \\
\hline & 300 & 2.71 & $2.28 \mathrm{e} 6$ & 7.63e3 & 103.0 & 0 & $5.17 \mathrm{e} 05$ & $1.89 \mathrm{e} 10$ \\
\hline & 100 & 1.57 & $5.24 \mathrm{e} 5$ & $5.29 \mathrm{e} 3$ & 30.5 & 0 & $1.33 \mathrm{e} 05$ & $5.91 \mathrm{e} 09$ \\
\hline & 50 & 2.26 & $2.01 \mathrm{e} 5$ & $4.09 \mathrm{e} 3$ & 15.5 & 0 & $6.04 \mathrm{e} 04$ & $3.07 \mathrm{e} 09$ \\
\hline & 10 & 0.04 & $1.50 \mathrm{e} 4$ & $1.67 \mathrm{e} 3$ & 3.3 & 0 & $3.00 \mathrm{e} 03$ & $9.64 \mathrm{e} 07$ \\
\hline PPSF & 2000 & 24.40 & $2.12 \mathrm{e} 7$ & $1.06 \mathrm{e} 4$ & 777.0 & 0 & $3.74 \mathrm{e} 06$ & $9.08 \mathrm{e} 10$ \\
\hline \multirow[t]{8}{*}{ CB3 II } & 1500 & 11.70 & $1.08 \mathrm{e} 7$ & $7.20 \mathrm{e} 3$ & 540.0 & 0 & $2.76 \mathrm{e} 06$ & $6.83 \mathrm{e} 10$ \\
\hline & 1000 & 5.62 & $5.35 \mathrm{e} 6$ & $5.36 \mathrm{e} 3$ & 341.0 & 0 & $1.84 \mathrm{e} 06$ & $2.67 \mathrm{e} 10$ \\
\hline & 800 & 4.14 & $3.98 \mathrm{e} 6$ & $4.98 \mathrm{e} 3$ & 261.0 & 0 & $1.47 \mathrm{e} 06$ & $3.80 \mathrm{e} 10$ \\
\hline & 500 & 2.24 & $2.17 \mathrm{e} 6$ & $4.35 \mathrm{e} 3$ & 163.0 & 0 & $9.04 \mathrm{e} 05$ & $2.08 \mathrm{e} 10$ \\
\hline & 300 & 1.29 & $1.31 \mathrm{e} 6$ & $4.36 \mathrm{e} 3$ & 97.1 & 0 & $5.08 \mathrm{e} 05$ & $1.93 \mathrm{e} 10$ \\
\hline & 100 & 0.86 & $9.97 \mathrm{e} 5$ & $1.01 \mathrm{e} 4$ & 29.8 & 0 & $1.36 \mathrm{e} 05$ & $3.98 \mathrm{e} 09$ \\
\hline & 50 & 0.25 & $1.99 \mathrm{e} 5$ & $4.05 \mathrm{e} 3$ & 15.6 & 0 & $7.76 \mathrm{e} 04$ & $2.09 \mathrm{e} 09$ \\
\hline & 10 & 0.02 & $1.08 \mathrm{e} 4$ & $1.19 \mathrm{e} 3$ & 3.4 & 0 & $3.68 \mathrm{e} 03$ & $4.93 \mathrm{e} 08$ \\
\hline \multirow{9}{*}{$\begin{array}{l}\text { PPSF } \\
\text { generalised } \\
\text { Brown } 2\end{array}$} & 2000 & 79.60 & $3.74 \mathrm{e} 7$ & $1.87 \mathrm{e} 4$ & 975.0 & 3 & $5.00 \mathrm{e} 02$ & $1.32 \mathrm{e} 03$ \\
\hline & 1500 & 39.80 & $1.94 \mathrm{e} 7$ & $1.30 \mathrm{e} 4$ & 724.0 & 0 & $4.01 \mathrm{e} 02$ & $9.97 \mathrm{e} 02$ \\
\hline & 1000 & 16.30 & $8.22 \mathrm{e} 6$ & $8.23 \mathrm{e} 3$ & 473.0 & 0 & $2.47 \mathrm{e} 02$ & $6.59 \mathrm{e} 02$ \\
\hline & 800 & 10.70 & $5.38 \mathrm{e} 6$ & $6.74 \mathrm{e} 3$ & 376.0 & 0 & $1.97 \mathrm{e} 02$ & $5.27 \mathrm{e} 02$ \\
\hline & 500 & 5.10 & $2.40 \mathrm{e} 6$ & $4.81 \mathrm{e} 3$ & 232.0 & 0 & $1.24 \mathrm{e} 02$ & $3.23 \mathrm{e} 02$ \\
\hline & 300 & 3.60 & $1.24 \mathrm{e} 6$ & $4.13 \mathrm{e} 3$ & 138.0 & 0 & $7.74 \mathrm{e} 01$ & $1.98 \mathrm{e} 02$ \\
\hline & 100 & 2.98 & $2.74 \mathrm{e} 5$ & $2.77 \mathrm{e} 3$ & 45.9 & 0 & $2.28 \mathrm{e} 01$ & $6.68 \mathrm{e} 01$ \\
\hline & 50 & 2.89 & $1.01 \mathrm{e} 5$ & $2.07 \mathrm{e} 3$ & 23.0 & 0 & $1.23 \mathrm{e} 01$ & $3.41 \mathrm{e} 01$ \\
\hline & 10 & 0.02 & $6.21 \mathrm{e} 3$ & $6.89 \mathrm{e} 2$ & 4.7 & 0 & $1.50 \mathrm{e} 00$ & $6.79 \mathrm{e} 00$ \\
\hline PPSF & 2000 & 54.50 & $6.18 \mathrm{e} 7$ & $3.09 \mathrm{e} 4$ & 525.0 & 93 & $1.25 \mathrm{e} 07$ & $2.21 \mathrm{e} 07$ \\
\hline \multirow[t]{8}{*}{ Broyden } & 1500 & 19.10 & $2.44 \mathrm{e} 7$ & $1.63 \mathrm{e} 4$ & 329.0 & 82 & $8.85 \mathrm{e} 06$ & $1.72 \mathrm{e} 07$ \\
\hline & 1000 & 8.41 & $1.16 \mathrm{e} 7$ & $1.16 \mathrm{e} 4$ & 199.0 & 32 & $6.25 \mathrm{e} 06$ & $1.10 \mathrm{e} 07$ \\
\hline & 800 & 5.59 & $7.99 \mathrm{e} 6$ & $1.00 \mathrm{e} 4$ & 164.0 & 8 & $5.05 \mathrm{e} 06$ & $8.75 \mathrm{e} 06$ \\
\hline & 500 & 2.48 & $3.32 \mathrm{e} 6$ & $6.66 \mathrm{e} 3$ & 109.0 & 0 & $2.62 \mathrm{e} 06$ & $5.73 \mathrm{e} 06$ \\
\hline & 300 & 1.32 & $1.58 \mathrm{e} 6$ & $5.29 \mathrm{e} 3$ & 70.9 & 0 & $1.75 \mathrm{e} 06$ & $3.35 \mathrm{e} 06$ \\
\hline & 100 & 0.57 & $2.96 \mathrm{e} 5$ & $2.99 \mathrm{e} 3$ & 26.5 & 0 & $5.27 \mathrm{e} 05$ & $1.36 \mathrm{e} 06$ \\
\hline & 50 & 0.62 & $1.26 \mathrm{e} 5$ & $2.56 \mathrm{e} 3$ & 14.0 & 0 & $2.39 \mathrm{e} 05$ & $6.85 \mathrm{e} 05$ \\
\hline & 10 & 0.06 & $1.60 \mathrm{e} 4$ & $1.78 \mathrm{e} 3$ & 3.1 & 0 & $1.05 \mathrm{e} 04$ & $1.95 \mathrm{e} 05$ \\
\hline
\end{tabular}

Table 3: Results for piecewise partially separable functions 


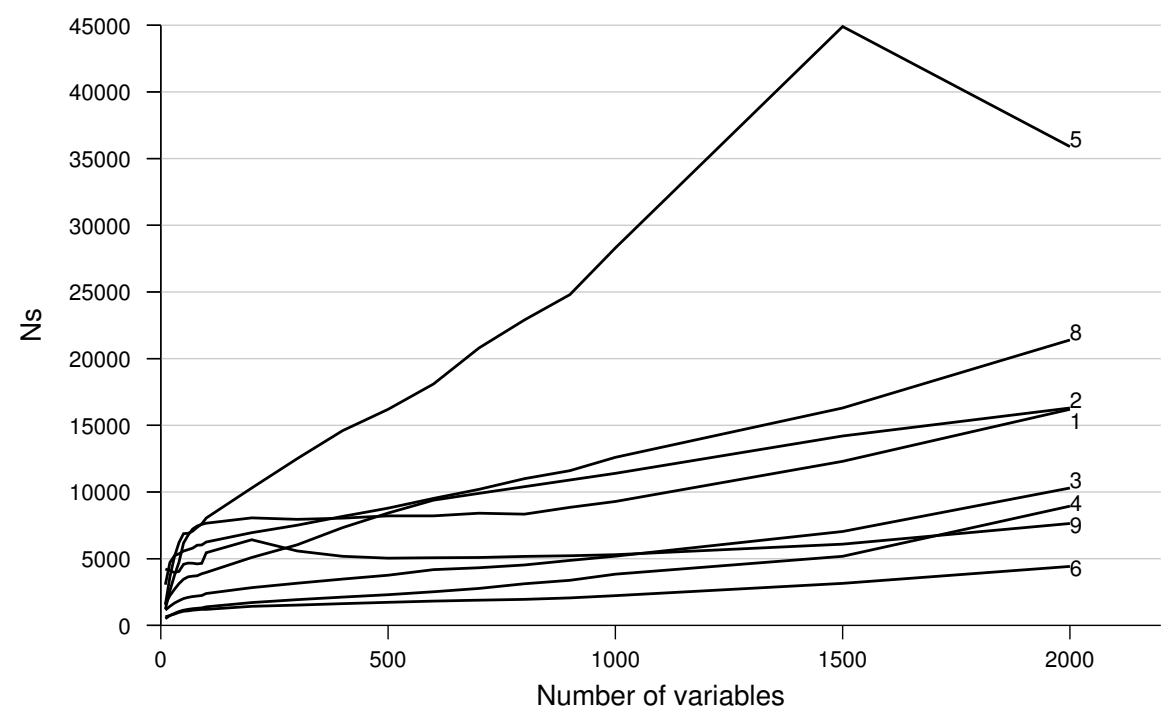

Figure 1: Average number of function evaluations for piecewise chained functions

As one can see from Tables 1 - 3 the proposed algorithm allows us to solve all problems with a given accuracy except Problem 4 (with $n=2000$ ), Problem 6 (with $n=1000,2000$ ), Problem 7 (with $n=100-2000$ ) and Problem 13 (with $n=800,1000,1500,2000$ ). However, it should be noted that all problems, except Problem 7, have been solved with rougher accuracy. In the numerical experiments we restricted the maximum number of discrete gradients which can be calculated at each iteration to 100. In all these problems in order to calculate solutions with higher accuracy we have to significantly increase this number. But in this case the CPU time may increase substantially.

Results for CPU time reported in the tables demonstrate that the algorithm is quite fast to find solutions with the given accuracy in problems up to 2000 variables.

The numbers presented in columns for the minimum and maximum values of the difference $f\left(x^{0}\right)-f_{*}$ show that randomly chosen initial points are not close to the solutions for all experiments. Therefore one can assert that the number of objective function evaluations $N_{S}$ is moderate for all problems and $n$. We can also see from Figures 1 and 3 that the number $N_{S}$ seems a linear function of the number of variables for all problems for which the algorithm was successful.

The ratio $N_{g} / N_{S}$ increases as the number of variables increases. Figures 2 and 4 demonstrate that this ratio is a linear function of the number of variables and $N_{g} / N_{S} \approx \alpha n$ where $\alpha=0.30 \div 0.95$.

The numerical results show that the number of term function evaluations is small in all cases, and therefore from a more practical viewpoint, almost all problems were solved in less than one minute on a normal PC. 


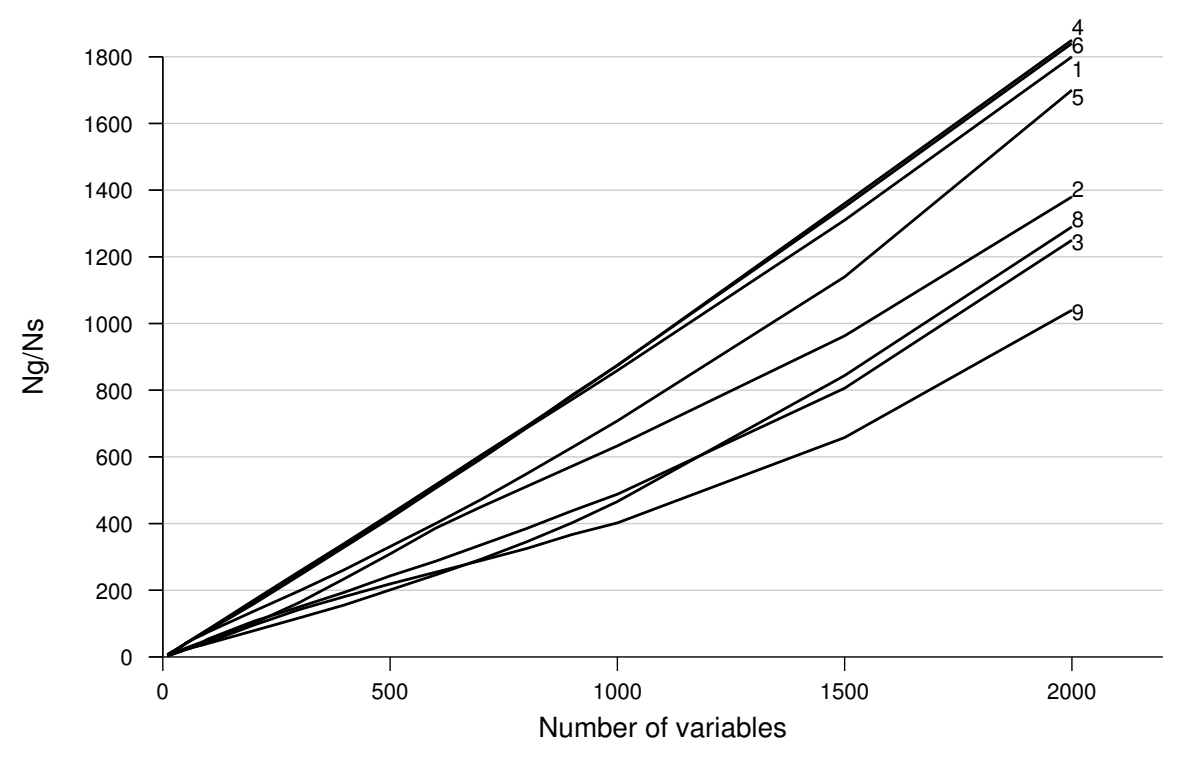

Figure 2: Average ratio of the number of function evaluations for general scheme to simplified scheme for piecewise chained functions

\section{Conclusions}

In this paper we have developed an algorithm for solving one class of large scale nonsmooth optimization. This class contains piecewise partially separable functions. These functions have many practical applications including applications in data mining and information retrieval. An algorithm for minimization of these functions is the modification of the discrete gradient method. It has been shown that the calculation of discrete gradients can be significantly accelerated. We present results of preliminary numerical experiments which demonstrate that the proposed algorithm is efficient for solving many large scale nonsmooth optimization problems up to 2000 variables.

As it was pointed out above in this paper the discrete gradient method consists of three major steps: the computation of the discrete gradients, the computation of a descent direction by solving a certain quadratic programming problem and a line search. The simplified scheme proposed in this paper allows one to significantly accelerate the computation of the discrete gradients. However, the acceleration of the two other steps taking into account the structure of problems may lead to more efficient algorithms to solve a broad class of large scale nonsmooth optimization problems. This will be the subject of our further research.

\section{Acknowledgements}

This research was supported by the Australian Research Council. 


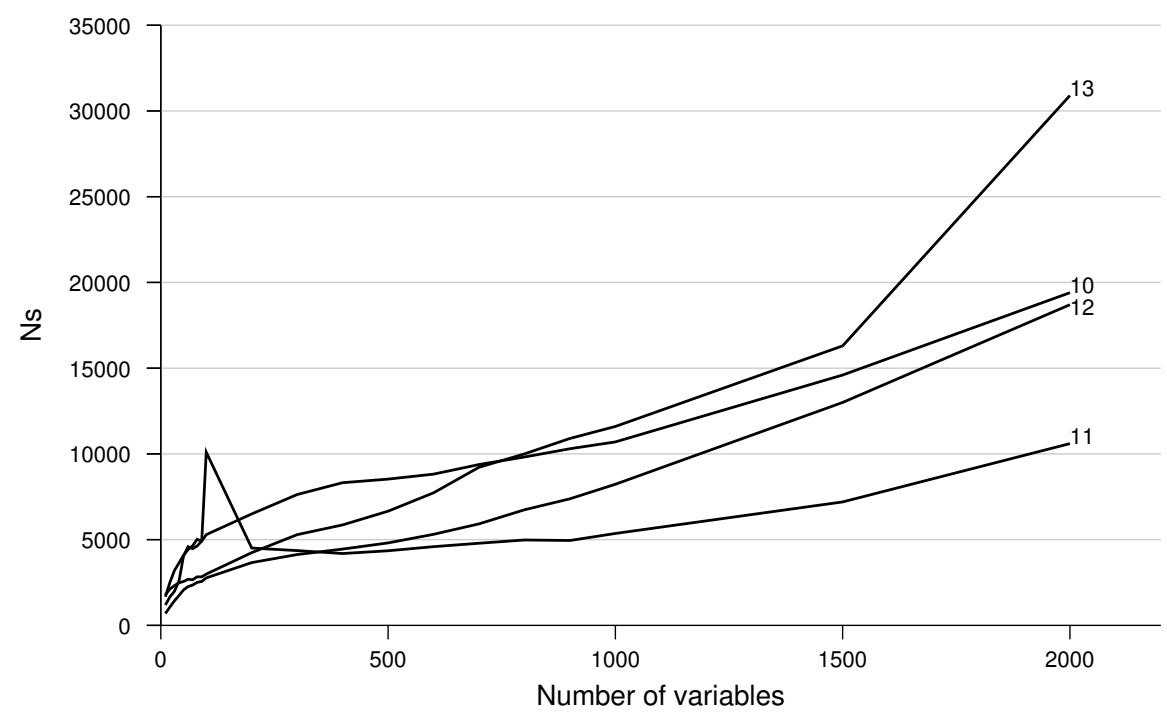

Figure 3: Average number of function evaluations for PPS functions

\section{References}

[1] B.M. Aberick, C.H. Bicshof, A. Carle, J. More and A. Griewank, Computing large sparse Jacobian matrices using automatic differentiation, SIAM Journal on Scientific and Statistical Computing, 15, 1994, 285-294.

[2] Armijo, L., Minimization of functions having continuous partial derivatives, Pacific J. Math., 16, 1966, 1-13.

[3] A.M. Bagirov and A.A. Gasanov, A method of approximating a quasidifferential, Russian Journal of Computational Mathematics and Mathematical Physics, 35(4), 1995, 403-409.

[4] A.M. Bagirov, Minimization methods for one class of nonsmooth functions and calculation of semi-equilibrium prices, In: A. Eberhard et al. (eds.) Progress in Optimization: Contribution from Australasia, Kluwer Academic Publishers, 1999, 147-175.

[5] A.M. Bagirov, A method for minimzation of quasidifferentiable functions, Optimization Methods and Software, 17(1), 2002, 31-60.

[6] A.M. Bagirov, Continuous subdifferential approximations and their applications, Journal of Mathematical Sciences, 115(5), 2003, 2567-2609.

[7] A.M. Bagirov, Max-min separability, to appear: Optmization Methods and Software. 


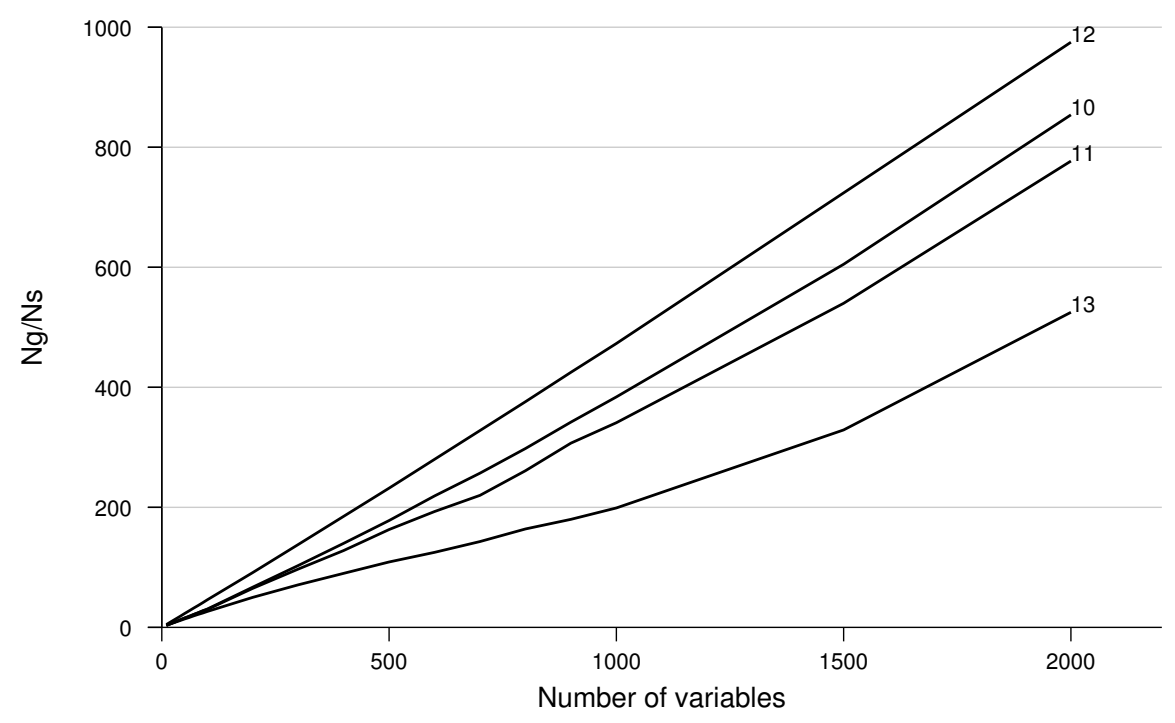

Figure 4: Average ratio of the number of function evaluations for general scheme to simplified scheme for PPS functions

[8] A.M. Bagirov, A.M. Rubinov, N.V. Soukhoroukova and J. Yearwood, Unsupervised and supervised data classification via nonsmooth and global optimization TOP: Spanish Journal of Operations Research, 2003, 1-93.

[9] A.M. Bagirov and J. Yearwood, A new nonsmooth optimization algorithm for minimum sum-of-squares clustering problems, to appear: European Journal of Operational Research.

[10] A.M. Bagirov and J. Ugon, An algorithm for minimizing clustering functions, Optimization, submitted.

[11] H.H. Bock, Automatische Klassifikation, Vandenhoeck \& Ruprecht, Gottingen, 1974.

[12] F.H. Clarke, Optimization and Nonsmooth Analysis, Wiley, New York, 1983.

[13] B. Colson and Ph.L. Toint, A derivative-free algorithm for sparse unconstrained optimization problems, in: A.H. Siddiqi and M. Kocvara (eds), Trends in Industrial and Applied Mathematics, 131-147, Kluwer Academic Publishers, Dordrecht, 2002.

[14] A.R. Conn, N. Gould and Ph.L. Toint, Improving the decomposition of partially separable functions in the context of large-scale optimization: a first approach, in W.W. Hager, D.W. Hearn and P.M. Pardalos (eds), Large Scale Optimization: State of the Art, 82-94, Kluwer Academic Publishers, Dordrecht, 1994. 
[15] V.F. Demyanov and A.M. Rubinov, Constructive Nonsmooth Analysis, Peter Lang, Frankfurt am Main, 1995.

[16] Yu.G. Evtushenko, A numerical method for finding best guaranteed estimates, USSR Journal of Computational Mathematics and Mathematical Physics, 12, 1972, 109128.

[17] A. Griewank and Ph.L. Toint, On the unconstrained optimization of partially separable functions, in: M.J.D. Powell (ed) Nonlinear Optimization, 301-312, Academic Press, 1982.

[18] M. Haarala, K. Miettinen and M.M. Makela, Large-scale nonsmooth optimization: New variable metric bundle algorithm with limited memory, to appear: Optimization Methods and Software.

[19] P. Hansen and B. Jaumard, Cluster analysis and mathematical programming, Mathematical Programming, 79(1-3), 1997, 191-215.

[20] J.-P. Hiriart-Urruty and C. Lemarechal, Convex Analysis and Minimization Algorithms, Vol. 1 and 2, Springer-Verlag, Berlin, New York, 1993.

[21] K.C. Kiwiel, Methods of descent for nondifferentiable optimization, Lecture Notes in Mathematics, 1133, Springer-Verlag, Berlin, 1985.

[22] A.K. Jain, M.N. Murty and P.J. Flynn, Data clustering: a review, ACM Computing Surveys 31(3), 1999, 264-323.

[23] L. Luksan and J. Vlcek, Sparse and partially separable test problems for unconstrained and equality constrained optimization, Technical Report 767, Institute of Computer Science, Academy of Sciences of the Czech Republic, Prague, 1999.

[24] L. Luksan and J. Vlcek, Test problems for nonsmooth unconstrained and linearly constrained optimization, Technical Report 798, Institute of Computer Science, Academy of Sciences of the Czech Republic, Prague, 2000.

[25] M.M. Makela and P. Neittaanmaki, Nonsmooth Optimization, World Scientific, Singapore, 1992.

[26] R. Mifflin, Semismooth and semiconvex functions in constrained optimization, SIAM Journal on Control and Optimization, 15(6), 1977, 959-972.

[27] J.A. Nelder and R. Mead, A simplex method for function minimization, Comput. J., 7, 1965, 308-313.

[28] M.J.D. Powell, UOBYQA: unconstrained optimization by quadratic approximation, Mathematical Programming, Series B, 92(3), 2002, 555-582. 